Research Article

\title{
Free Vibration Analysis of Functionally Graded Spherical Torus Structure with Uniform Variable Thickness along Axial Direction
}

\author{
Cong Gao $\mathbb{D}^{1},{ }^{1}$ Xuhong Miao $\mathbb{D D}^{1,2}$ Lin Lu ${ }^{1},{ }^{1}$ Ruidong Huo, ${ }^{1}$ Qiaolin Hu ${ }^{1}{ }^{3}$ \\ and Yanhe Shan $\mathbb{D I D}^{1}$ \\ ${ }^{1}$ College of Shipbuilding Engineering, Harbin Engineering University, Harbin 150001, China \\ ${ }^{2}$ Naval Research Academy, Beijing 100161, China \\ ${ }^{3}$ Jinan Vocational College, Jinan 250014, China \\ Correspondence should be addressed to Xuhong Miao; miaoxuhong@aliyun.com and Qiaolin Hu; hu009@126.com
}

Received 2 July 2019; Revised 5 September 2019; Accepted 14 September 2019; Published 11 November 2019

Academic Editor: Giorgio Dalpiaz

Copyright (c) 2019 Cong Gao et al. This is an open access article distributed under the Creative Commons Attribution License, which permits unrestricted use, distribution, and reproduction in any medium, provided the original work is properly cited.

Based on the Ritz method, this paper focused on the free vibration of functionally graded (FG) spherical torus with uniform variable thickness along axial direction under different boundary conditions. The first-order shear deformation theory (FSDT) is employed to formulate the analytical model. The method involves partitioning of the spherical torus structure into proper shell segments in order to satisfy the computing requirement of high-order vibration responses according to the domain decomposition method. The two adjacent segments are connected by using the penalty method, where penalty parameters are defined by the artificial springs; the continuity condition and different boundary conditions can be obtained by assigning the appropriate values of springs. The displacement functions' components are double mixed series, in which Fourier series and unified Jacobi polynomials, respectively, represent displacement function along circumferential direction and axial direction. Then the Ritz method is used to obtain final solutions. The numerical results obtained by the proposed method show great agreement with previously published literatures and those from the finite element program ABAQUS. The effects of boundary conditions and geometric parameters on the vibration responses of the structure are also presented. The most novelty of this paper is to generalize the selection of admissible displacement functions by using Jacobi polynomial.

\section{Introduction}

The functionally graded material has been widely utilized to make up various shells since the conception of it was first presented in 1984 [1], the FG spherical torus structures occupy an important position in practical engineering fields such as architecture and machine due to their excellent mechanical properties. In practical applications, these shell structures are usually subject to dynamic loads which may cause vibration and structural damage unless a sufficient vibration characteristic research has been done at the design stage. Furthermore, the boundary restraints of the variable thickness FG spherical torus are often abundant and diverse in actual working process. According to this background, the main purpose of the research is to propose a unified solution formulation to study the free vibration characteristics of FG spherical torus with uniform variable thickness along axial direction under general boundary restraints.
Much attention has been devoted over the years to investigate the dynamic characteristics of spherical shell structures, and a large number of numerical and analytical approaches have been presented to carry out the vibration behaviors from the existing literatures, such as the generalized differential quadrature (GDQ) method [2-5], the Ritz method [6-9], the separation of variables method $[10,11]$, and the finite element method (FEM) [12-17]. In addition, many studies have been focused to obtain the vibration solutions of FG spherical shell. Su et al. [18] put forward an accurate formulation to study the free vibration of FG spherical shell structures subjected to general edge restraints according to FSDT, and the admissible functions consist of standard Fourier cosine series. Wang et al. $[19,20]$ demonstrated the application of Ritz method for vibration analysis of FG shell structures under different edge restraints. On the assumption that the material properties vary consecutively along the direction of shell thickness, $\mathrm{Qu}$ et al. 
[21] proposed a unified solution to analyze the free vibration of FG spherical shells under arbitrary boundaries based on the FSDT. Li et al. [22-24] used a semianalytical method to analyze the free vibration characteristics of FG shells of revolution. Tomabene and Viola [25] carried out a great deal of studies on the vibration solutions of FG spherical shell structures by applying the GDQ method. Considering the stretching and predominantly flexural vibration, Reddy and Cheng [26] discussed the effects on natural frequencies of FG spherical shallow shell by applying the classical theory and the first-order and third-order shear deformation theories. In the framework of the higher-order shear deformation theory (HSDT), Neves et al. [27] investigated the free vibration behaviors of FG shell structures, in which the expressions of motion and the boundary restraints were acquired by Carrera's unified formulation. Kar and Panda [28] analyzed the vibration characteristics of FG spherical shell structure based on the HSDT, and the analytical model is discretized by using quadrilateral Lagrangian element.

Some literatures relevant to the studies on the vibration characteristics of variable thickness shell structures are as follows. Based on Flugge thin shell theory, El-Kaabazi and Kennedy [29] predicted the vibration frequencies and mode shapes of variable thickness cylindrical shells by applying the dynamic stiffness method (DQM). The transfer matrix method was proposed by Liu et al. [30] to analyze the vibration responses of conical shell with variable thickness. Qu et al. [31, 32] put forward a domain decomposition method to investigate the vibration behaviors of stepped shell structures with various step numbers. Considering two sets of edge restraints, Wei et al. [33] applied the Rayleigh-Ritz method to calculate the vibration solutions of the stiffened hollow conical shell structures with different variable thickness distribution modes. Kang and Leissa [34] analyzed the free vibration characteristics of shell structures of revolution with variable thickness by using Ritz method. According to GDQ method, Xiang and Yang [35] performed the vibration characteristics of FG beam with variable thickness, and the free vibration characteristics of variable thickness shells were systematically studied by Jiang and Redekop [36]. Nejad et al. [37] reported the vibration characteristics of functionally graded cylinder shells with variable thickness by applying the FEM method. More detailed descriptions about vibration behaviors of related structure can be found in references [38-41].

Through the aforementioned literature review, it can be easily concluded that the current numerical and analytical methods are abundant to analyze the vibration behaviors of uniform isotropic spherical shells, FG spherical shells, and stepped shell structures under various boundary conditions. However, the existing literature focused on free vibration behaviors of FG spherical torus with uniform variable thickness under general edge conditions are limited, and most of the current studies concentrated on the free and forced vibration of plate or beam structures with uniform variable thickness. Based on this background, it is of great significance to carry out the vibration solutions of FG spherical torus structures with uniform variable thickness subject to general edge conditions. The numerical results obtained by the proposed method show great agreement with previously published literatures and those from the finite element program ABAQUS. The effects of some geometrical parameters on the free vibration behaviors of FG spherical torus with uniform variable thickness along axial direction are also investigated. The novelty of this paper is the unified Jacobi-Ritz formulation to study the free vibration characteristics of stepped FG spherical torus shell with general boundary conditions, and the selection of admissible displacement functions are generalized by using the Jacobi polynomial.

\section{Mathematical Formulation}

2.1. The Mathematical Model of Uniform Variable Thickness FG Spherical Torus. The mathematical model of uniform variable thickness FG spherical torus is depicted in Figure 1. $R$ and $C_{s}$ denote radius and geometry center of the spherical torus structures, respectively. $\theta_{0}$ and $\theta_{\zeta}$ represent the center angle correspond to the top and bottom of uniform variable thickness FG spherical torus, accordingly. $h_{0}$ denotes the thickness of the top structure that related to $\theta_{0}$, while $h_{i}$ represents the thickness of the $i$ th segment related to $\theta_{i}$, and the relationship between $h_{i}$ and $h_{0}$ is $h_{i}=h_{0}+\left(\theta_{i}-\theta_{0}\right) h_{0} / \pi$. The system $(\theta, \varphi, \delta)$ is adopted to describe the spherical torus, and respectively represent the axial, circumferential, and normal directions. In addition, the displacements of $\theta$, $\varphi$, and $\delta$ are, respectively, represented by $u, v$, and $w$. As Figure 1 shows, the spherical torus is divided into $\zeta$ segments along axial direction, and five groups of artificial springs $\left(k_{u}\right.$, $\left.k_{v}, k_{w}, k_{\theta}, k_{\varphi}\right)$ are set at both ends of each segment. The continuity condition and the given edge restraints can be simulate by adjusting spring stiffness.

Two kinds of the FG model are considered in this paper, and the essential material parameters of the FG spherical torus are expressed as below:

$$
\begin{aligned}
& E(\delta)=\left(E_{1}-E_{2}\right) V_{1}+E_{2}, \\
& \mu(\delta)=\left(\mu_{1}-\mu_{2}\right) V_{1}+\mu_{2}, \\
& \rho(\delta)=\left(\rho_{1}-\rho_{2}\right) V_{1}+\rho_{2},
\end{aligned}
$$

where $E_{1}, \rho_{1}, \mu_{1}$ and $E_{2}, \rho_{2}, \mu_{2}$, respectively, represent Young's modulus, mass density and Poisson's ratios of the ceramic and metallic constituents, and the volume fraction of the ceramic is denoted by $V_{1}$. In present study, two general four-parameter power-law distributions are shown in $[42,43]$ :

$$
\begin{aligned}
& \mathrm{FGM}_{\mathrm{I}(a / b / c / p)}: V_{1}=\left[1-a\left(\frac{1}{2}+\frac{\delta}{h}\right)+b\left(\frac{1}{2}+\frac{\delta}{h}\right)^{c}\right]^{p}, \\
& \mathrm{FGM}_{\mathrm{II}(a / b / c / p)}: V_{1}=\left[1-a\left(\frac{1}{2}-\frac{\delta}{h}\right)+b\left(\frac{1}{2}-\frac{\delta}{h}\right)^{c}\right]^{p},
\end{aligned}
$$

where $p(p \geq 0)$ is the power-law exponent. The variation of parameters $a, b$, and $c$ can decide the different power-law distributions. 


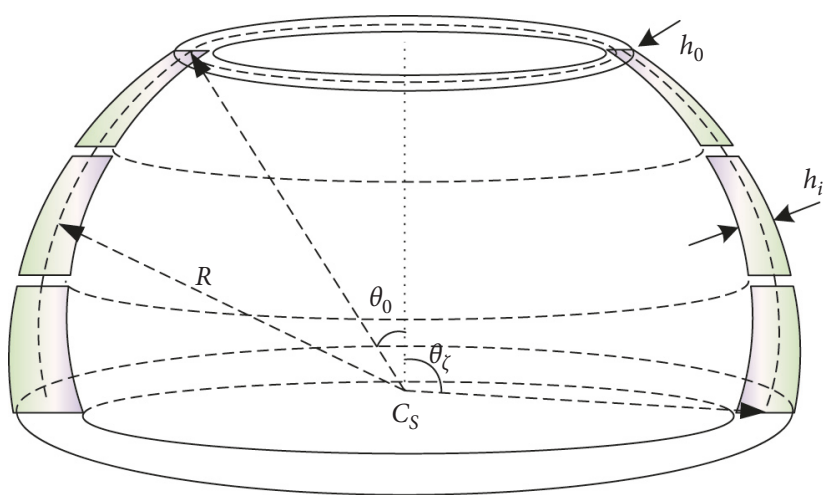

(a)

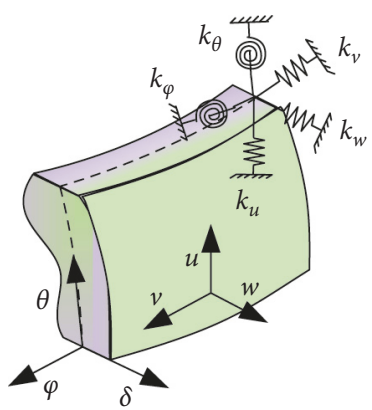

(b)

FIGURE 1: Geometry notations and coordinate system of FG spherical shell with uniform variable thickness.

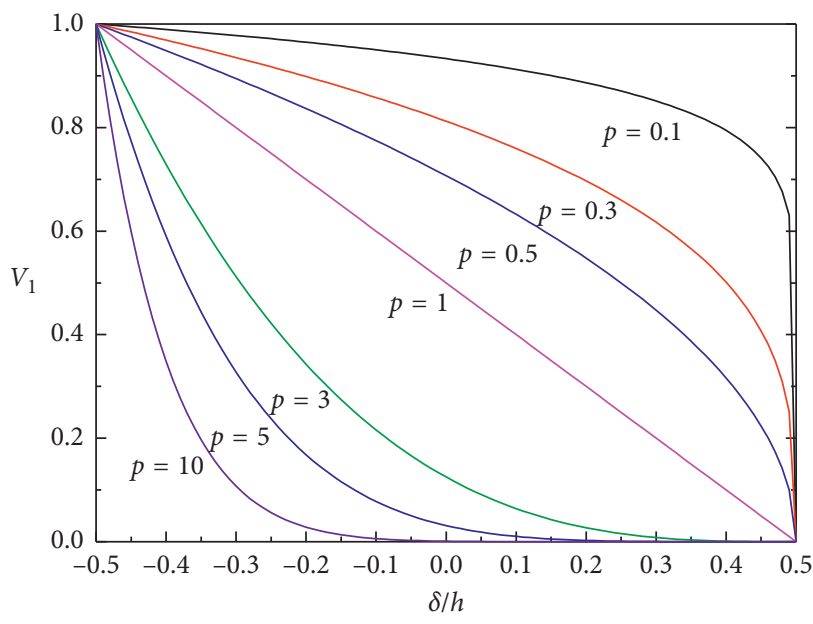

(a)

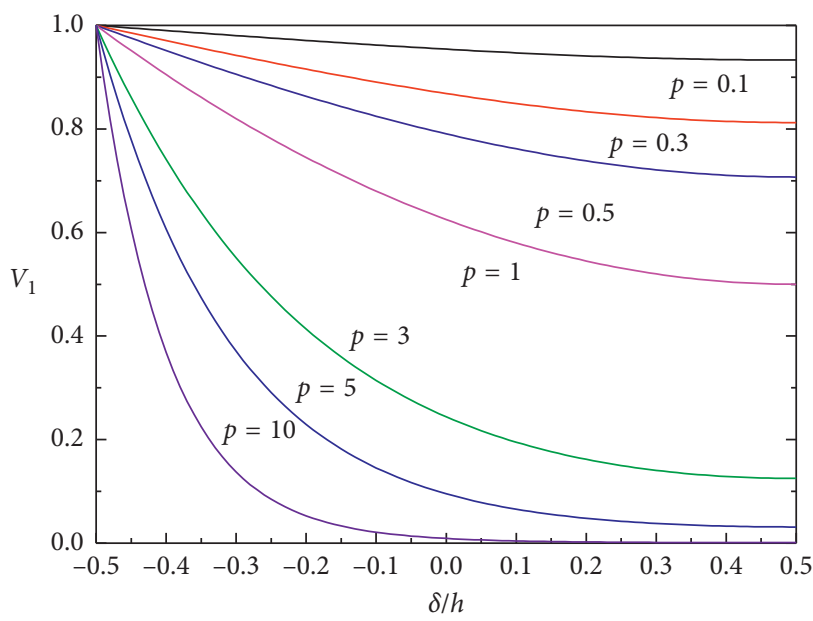

(c)

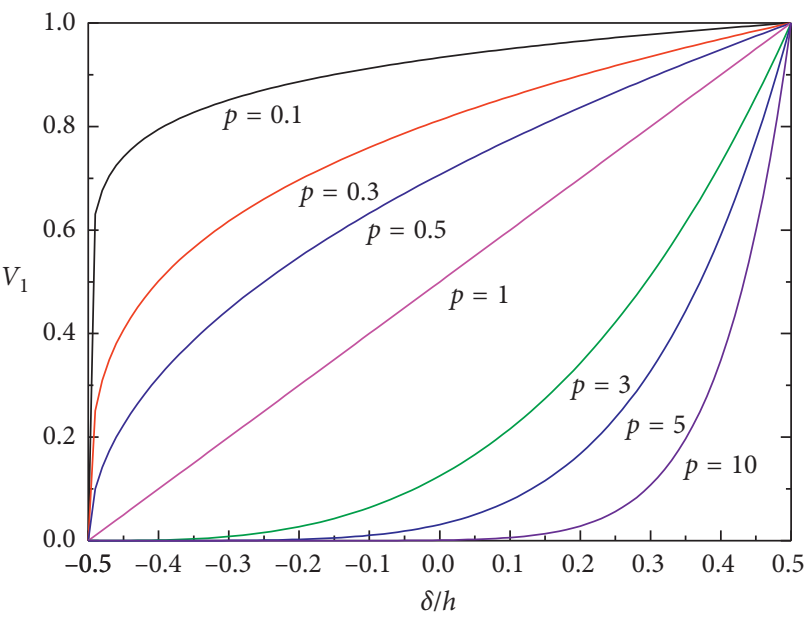

(b)

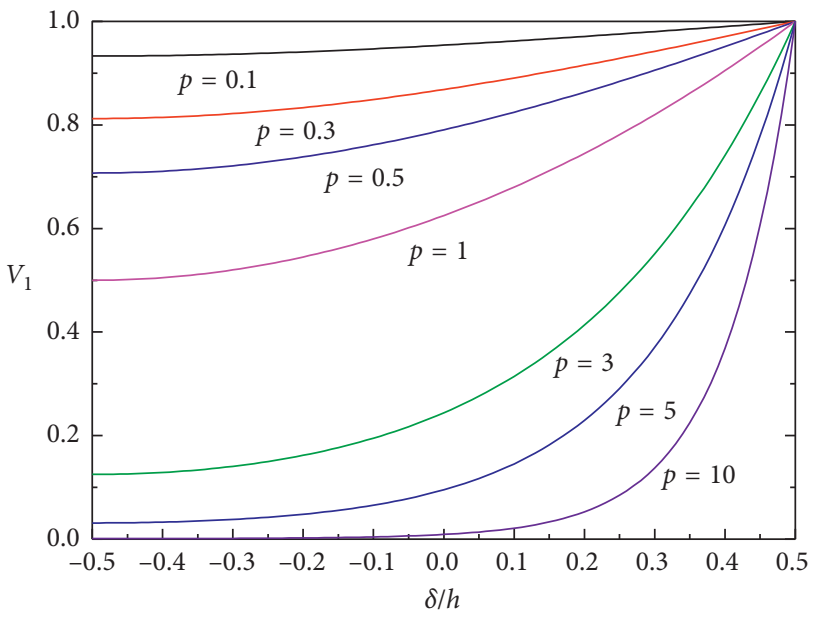

(d)

Figure 2: Variations of the volume fraction $\left(V_{1}\right)$ through the structure thickness for different values of power-law exponent $p$. (a) $\operatorname{FGM}_{\mathrm{I}(a=1 / b=0 / c / p)}$. (b) $\operatorname{FGM}_{\mathrm{II}(a=1 / b=0 / c / p)}$. (c) $\operatorname{FGM}_{\mathrm{I}(a=1 / b=0.5 / c=2 / p)}$. (d) $\operatorname{FGM}_{\mathrm{II}(a=1 / b=0.5 / c=2 / p)}$.

Figure 2 shows the variations of the volume fraction through the structure thickness for different values of power-law exponent $p$. It is obvious that the two type distributions are symmetrical. In addition, the isotropic material can be obtained as a particular case when the value of $p$ is set as infinity or zero. 
2.2. Expressions of Uniform Variable Thickness FG Spherical Torus's Energy. In this paper, the FSDT [44-46] theory is adopted to describe the displacements of the $i$ th segment of the uniform variable thickness FG spherical torus:

$$
\begin{aligned}
\bar{U}^{i}(\theta, \varphi, \delta, t) & =u^{i}(\theta, \varphi, t)+\delta \psi_{\theta}^{i}(\theta, \varphi, t), \\
\bar{V}^{i}(\theta, \varphi, \delta, t) & =v^{i}(\theta, \varphi, t)+\delta \psi_{\varphi}^{i}(\theta, \varphi, t), \\
\bar{W}^{i}(\theta, \varphi, \delta, t) & =w^{i}(\theta, \varphi, t),
\end{aligned}
$$

where the rotation of transverse normal are represented by $\psi_{\theta}^{i}$ and $\psi_{\varphi}^{i}$, respectively.

The strains of the shell structure in this paper can be written as

$$
\begin{aligned}
\varepsilon_{\theta}^{i} & =\varepsilon_{\theta}^{0, i}+\delta \kappa_{\theta}^{i}, \\
\varepsilon_{\varphi}^{i} & =\varepsilon_{\varphi}^{0, i}+\delta \kappa_{\varphi}^{i}, \\
\gamma_{\theta \varphi}^{i} & =\gamma_{\theta \varphi}^{0, i}+\delta \kappa_{\theta \varphi}^{i}, \\
\gamma_{\theta \delta}^{i} & =\gamma_{\theta \delta}^{0, i}, \\
\gamma_{\varphi \delta}^{i} & =\gamma_{\varphi \delta}^{0, i},
\end{aligned}
$$

where $\varepsilon_{\theta}^{i}, \varepsilon_{\varphi}^{i}, \gamma_{\varphi \theta}^{i}, \kappa_{\theta}^{i}, \kappa_{\varphi}^{i}, \kappa_{\varphi \theta}^{i}, \gamma_{\theta \delta}^{0, i}$ and $\gamma_{\varphi \delta}^{0, i}$ can be, respectively, expressed as

$$
\begin{aligned}
\varepsilon_{\theta}^{0, i} & =\frac{1}{A} \frac{\partial u^{i}}{\partial \theta}+\frac{v^{i}}{A B} \frac{\partial A}{\partial \varphi}+\frac{w^{i}}{R}, \\
\varepsilon_{\varphi}^{0, i} & =\frac{1}{B} \frac{\partial v^{i}}{\partial \varphi}+\frac{u^{i}}{A B} \frac{\partial B}{\partial \theta}+\frac{w^{i}}{R}, \\
\gamma_{\theta \varphi}^{0, i} & =\frac{A}{B} \frac{\partial}{\partial \varphi}\left(\frac{u^{i}}{A}\right)+\frac{B}{A} \frac{\partial}{\partial \theta}\left(\frac{v^{i}}{B}\right), \\
\kappa_{\theta}^{i} & =\frac{1}{A} \frac{\partial \psi_{\theta}^{i}}{\partial \theta}+\frac{\psi_{\varphi}^{i}}{A B} \frac{\partial A}{\partial \varphi}, \\
\kappa_{\varphi}^{i} & =\frac{1}{B} \frac{\partial \psi_{\varphi}^{i}}{\partial \varphi}+\frac{\psi_{\theta}^{i}}{A B} \frac{\partial B}{\partial \theta}, \\
\kappa_{\theta \varphi}^{i} & =\frac{A}{B} \frac{\partial}{\partial \varphi}\left(\frac{\psi_{\theta}^{i}}{A}\right)+\frac{B}{A} \frac{\partial}{\partial \theta}\left(\frac{\psi_{\varphi}^{i}}{B}\right), \\
\gamma_{\theta \delta}^{0, i} & =\frac{1}{A} \frac{\partial w^{i}}{\partial \theta}-\frac{u^{i}}{R}+\psi_{\theta}^{i}, \\
\gamma_{\varphi \delta}^{0, i} & =\frac{1}{B} \frac{\partial w^{i}}{\partial \varphi}-\frac{v^{i}}{R}+\psi_{\varphi}^{i} .
\end{aligned}
$$

For uniform variable thickness FG shell in this paper, the Lamé parameters $\mathrm{A}$ and $\mathrm{B}$ are shown as

$$
\begin{aligned}
& A=R, \\
& B=R \sin \varphi,
\end{aligned}
$$

Then the stresses mentioned above can be written as

$$
\left\{\begin{array}{c}
\sigma_{\theta}^{i} \\
\sigma_{\varphi}^{i} \\
\tau_{\theta \varphi}^{i} \\
\tau_{\theta \delta}^{i} \\
\tau_{\varphi \delta}^{i}
\end{array}\right\}=\left[\begin{array}{ccccc}
Q_{11}(\delta) & Q_{12}(\delta) & 0 & 0 & 0 \\
Q_{12}(\delta) & Q_{22}(\delta) & 0 & 0 & 0 \\
0 & 0 & Q_{66}(\delta) & 0 & 0 \\
0 & 0 & 0 & Q_{66}(\delta) & 0 \\
0 & 0 & 0 & 0 & Q_{66}(\delta)
\end{array}\right]\left\{\begin{array}{c}
\varepsilon_{\theta}^{i} \\
\varepsilon_{\varphi}^{i} \\
\gamma_{\theta \varphi}^{i} \\
\gamma_{\theta \delta}^{i} \\
\gamma_{\varphi \delta}^{i}
\end{array}\right\},
$$

where the symbols of $\sigma$ and $\tau$, respectively, represent normal and shear stresses. The $Q_{i j}(\delta)(i, j=1,2,6)$ are defined as follows:

$$
\begin{aligned}
Q_{11}(\delta)=Q_{22}(\delta) & =\frac{E(\delta)}{1-\mu^{2}(\delta)}, \\
Q_{12}(\delta) & =\frac{\mu(\delta) E(\delta)}{1-\mu^{2}(\delta)}, \\
Q_{66}(\delta) & =\frac{E(\delta)}{2[1+\mu(\delta)]},
\end{aligned}
$$

Based on the stress integral principle, the force and moment resultants are shown as

$$
\begin{gathered}
\left\{\begin{array}{c}
N_{\theta}^{i} \\
N_{\varphi}^{i} \\
N_{\theta \varphi}^{i}
\end{array}\right\}=\left[\begin{array}{ccc}
A_{11} & A_{12} & 0 \\
A_{12} & A_{22} & 0 \\
0 & 0 & A_{66}
\end{array}\right]\left\{\begin{array}{c}
\varepsilon_{\theta}^{0, i} \\
\varepsilon_{\varphi}^{0, i} \\
\gamma_{\theta \varphi}^{0, i}
\end{array}\right\}+\left[\begin{array}{ccc}
B_{11} & B_{12} & 0 \\
B_{12} & B_{22} & 0 \\
0 & 0 & B_{66}
\end{array}\right]\left\{\begin{array}{c}
\kappa_{\theta}^{i} \\
\kappa_{\varphi}^{i} \\
\kappa_{\theta \varphi}^{i}
\end{array}\right\}, \\
\left\{\begin{array}{c}
M_{\theta}^{i} \\
M_{\varphi}^{i} \\
M_{\theta \varphi}^{i}
\end{array}\right\}=\left[\begin{array}{ccc}
B_{11} & B_{12} & 0 \\
B_{12} & B_{22} & 0 \\
0 & 0 & B_{66}
\end{array}\right]\left\{\begin{array}{c}
\varepsilon_{\theta}^{0, i} \\
\varepsilon_{\varphi}^{0, i} \\
\gamma_{\theta \varphi}^{0, i}
\end{array}\right\}+\left[\begin{array}{ccc}
D_{11} & D_{12} & 0 \\
D_{12} & D_{22} & 0 \\
0 & 0 & D_{66}
\end{array}\right]\left\{\begin{array}{c}
\kappa_{\theta}^{i} \\
\kappa_{\varphi}^{i} \\
\kappa_{\theta \varphi}^{i}
\end{array}\right\},
\end{gathered}
$$

$$
\left\{\begin{array}{c}
Q_{\theta}^{i} \\
Q_{\varphi}^{i}
\end{array}\right\}=\bar{\kappa}\left[\begin{array}{cc}
A_{66} & 0 \\
0 & A_{66}
\end{array}\right]\left[\begin{array}{c}
\gamma_{\theta \delta}^{0, i} \\
\gamma_{\varphi \delta}^{0, i}
\end{array}\right]
$$

where $\bar{\kappa}$ is shear correction factor, and it is assumed to the value of 5/6 in the study. The symbols of $A_{i j}, B_{i j}$, and $D_{i j}$ can be obtained by the following formula:

$$
\left(A_{i j}, B_{i j}, D_{i j}\right)=\int_{-h / 2}^{h / 2} Q_{i j}(\delta)\left(1, \delta, \delta^{2}\right) \mathrm{d} \delta .
$$

The strain energy of the select segment for uniform variable thickness FG spherical torus can be expressed from equation (11):

$$
\begin{aligned}
U^{i}= & \frac{1}{2} \iiint_{V}\left(N_{\theta}^{i} \varepsilon_{\theta}^{0, i}+N_{\varphi}^{i} \varepsilon_{\varphi}^{0, i}+N_{\theta \varphi}^{i} \gamma_{\theta \varphi}^{0, i}+M_{\theta}^{i} k_{\theta}^{i}\right. \\
& \left.+M_{\varphi}^{i} k_{\varphi}^{i}+M_{\theta \varphi}^{i} k_{\theta \varphi}^{i}+Q_{\theta}^{i} \gamma_{\theta \delta}^{0, i}+Q_{\varphi}^{i} \gamma_{\varphi \delta}^{0, i}\right) A B \mathrm{~d} \theta \mathrm{d} \varphi \mathrm{d} \delta .
\end{aligned}
$$

To better represent the relationships between equation (11) and displacements, the strain energy can be expressed as 
$U^{i}=U_{S}^{i}+U_{B}^{i}+U_{B C}^{i}$, in which the detailed expressions can be written as

$$
\begin{aligned}
& U_{S}^{i}=\frac{1}{2} \iiint_{V}\left\{A_{11}\left(\frac{1}{A} \frac{\partial u^{i}}{\partial \theta}+\frac{v^{i}}{A B} \frac{\partial A}{\partial \varphi}+\frac{w^{i}}{R}\right)^{2}\right. \\
& +A_{22}\left(\frac{1}{B} \frac{\partial v^{i}}{\partial \varphi}+\frac{u^{i}}{A B} \frac{\partial B}{\partial \theta}+\frac{w^{i}}{R}\right)^{2} \\
& +A_{66}\left(\frac{A}{B} \frac{\partial}{\partial \varphi}\left(\frac{u^{i}}{A}\right)+\frac{B}{A} \frac{\partial}{\partial \theta}\left(\frac{v^{i}}{B}\right)\right)^{2} \\
& +2 A_{12}\left(\frac{1}{A} \frac{\partial u^{i}}{\partial \theta}+\frac{v^{i}}{A B} \frac{\partial A}{\partial \varphi}+\frac{w^{i}}{R}\right) \\
& \cdot\left(\frac{1}{B} \frac{\partial v^{i}}{\partial \varphi}+\frac{u^{i}}{A B} \frac{\partial B}{\partial \theta}+\frac{w^{i}}{R}\right)+\bar{\kappa} A_{66}\left(\frac{1}{A} \frac{\partial w^{i}}{\partial \theta}-\frac{u^{i}}{R}+\psi_{\theta}^{i}\right)^{2} \\
& \left.+\bar{\kappa} A_{66}\left(\frac{1}{B} \frac{\partial w^{i}}{\partial \varphi}-\frac{v^{i}}{R}+\psi_{\varphi}^{i}\right)^{2}\right\} A B \mathrm{~d} \theta \mathrm{d} \varphi \mathrm{d} \delta, \\
& U_{B}^{i}=\frac{1}{2} \iiint_{V}\left\{D_{11}\left(\frac{1}{A} \frac{\partial \psi_{\theta}^{i}}{\partial \theta}+\frac{\psi_{\varphi}^{i}}{A B} \frac{\partial A}{\partial \varphi}\right)^{2}\right. \\
& +D_{22}\left(\frac{1}{B} \frac{\partial \psi_{\varphi}^{i}}{\partial \varphi}+\frac{\psi_{\theta}^{i}}{A B} \frac{\partial B}{\partial \theta}\right)^{2} \\
& +D_{66}\left(\frac{A}{B} \frac{\partial}{\partial \varphi}\left(\frac{\psi_{\theta}^{i}}{A}\right)+\frac{B}{A} \frac{\partial}{\partial \theta}\left(\frac{\psi_{\varphi}^{i}}{B}\right)\right)^{2} \\
& +2 D_{12}\left(\frac{1}{A} \frac{\partial \psi_{\theta}^{i}}{\partial \theta}+\frac{\psi_{\varphi}^{i}}{A B} \frac{\partial A}{\partial \varphi}\right) \\
& \left.\cdot\left(\frac{1}{B} \frac{\partial \psi_{\varphi}^{i}}{\partial \varphi}+\frac{\psi_{\theta}^{i}}{A B} \frac{\partial B}{\partial \theta}\right)\right\} A B \mathrm{~d} \theta \mathrm{d} \varphi \mathrm{d} \delta \\
& U_{B S}^{i}=\iiint_{V}\left\{B_{11}\left(\frac{1}{A} \frac{\partial u^{i}}{\partial \theta}+\frac{v^{i}}{A B} \frac{\partial A}{\partial \varphi}+\frac{w^{i}}{R}\right)\left(\frac{1}{A} \frac{\partial \psi_{\theta}^{i}}{\partial \theta}+\frac{\psi_{\varphi}^{i}}{A B} \frac{\partial A}{\partial \varphi}\right)\right. \\
& +B_{12}\left(\frac{1}{A} \frac{\partial u^{i}}{\partial \theta}+\frac{v^{i}}{A B} \frac{\partial A}{\partial \varphi}+\frac{w^{i}}{R}\right)\left(\frac{1}{B} \frac{\partial \psi_{\varphi}^{i}}{\partial \varphi}+\frac{\psi_{\theta}^{i}}{A B} \frac{\partial B}{\partial \theta}\right) \\
& +B_{12}\left(\frac{1}{B} \frac{\partial v^{i}}{\partial \varphi}+\frac{u^{i}}{A B} \frac{\partial B}{\partial \theta}+\frac{w^{i}}{R}\right)\left(\frac{1}{A} \frac{\partial \psi_{\theta}^{i}}{\partial \theta}+\frac{\psi_{\varphi}^{i}}{A B} \frac{\partial A}{\partial \varphi}\right) \\
& +B_{66}\left(\frac{1}{A} \frac{\partial u^{i}}{\partial \theta}+\frac{v^{i}}{A B} \frac{\partial A}{\partial \varphi}+\frac{w^{i}}{R}\right)\left(\frac{A}{B} \frac{\partial}{\partial \varphi}\left(\frac{\psi_{\theta}^{i}}{A}\right)+\frac{B}{A} \frac{\partial}{\partial \theta}\left(\frac{\psi_{\varphi}^{i}}{B}\right)\right) \\
& \left.+B_{22}\left(\frac{1}{B} \frac{\partial \nu^{i}}{\partial \varphi}+\frac{u^{i}}{A B} \frac{\partial B}{\partial \theta}+\frac{w^{i}}{R}\right)\left(\frac{1}{B} \frac{\partial \psi_{\varphi}^{i}}{\partial \varphi}+\frac{\psi_{\theta}^{i}}{A B} \frac{\partial B}{\partial \theta}\right)\right\} A B \mathrm{~d} \theta \mathrm{d} \varphi \mathrm{d} \delta,
\end{aligned}
$$

where $U_{S}^{i}, U_{B}^{i}$, and $U_{B C}^{i}$ can be, respectively, clarified by $A_{i j}$, $D_{i j}$, and $B_{i j}$ according to equation (10).

The kinetic energy of the select segment can be shown as

$$
T^{i}=\frac{1}{2} \iiint_{V} \rho(\delta)\left[\left(\dot{\bar{U}}^{i}\right)^{2}+\left(\dot{\bar{V}}^{i}\right)^{2}+\left(\dot{\bar{W}}^{i}\right)^{2}\right]\left(1+\frac{\delta}{R}\right)^{2}
$$

$A B \mathrm{~d} \theta \mathrm{d} \varphi \mathrm{d} \delta$

$$
\begin{aligned}
= & \frac{1}{2} \int_{\theta_{0}}^{\theta \varsigma} \int_{0}^{2 \pi}\left\{I_{0}\left[\left(\dot{u}^{i}\right)^{2}+\left(\dot{v}^{i}\right)^{2}+\left(\dot{w}^{i}\right)^{2}\right]\right. \\
& \left.+2 I_{1}\left(\dot{u}^{i} \dot{\psi}_{\theta}^{i}+\dot{v}^{i} \dot{\psi}_{\varphi}^{i}\right)+I_{2}\left[\left(\dot{\psi}_{\theta}^{i}\right)^{2}+\left(\dot{\psi}_{\varphi}^{i}\right)^{2}\right]\right\} A B \mathrm{~d} \theta \mathrm{d} \varphi,
\end{aligned}
$$

where the dot on the symbols denote the differentiation about time, and $I_{0}, I_{1}$, and $I_{2}$ can be obtained by the following formula:

$$
\left(I_{0}, I_{1}, I_{2}\right)=\int_{-h / 2}^{h / 2} \rho(\delta)\left(1+\frac{\delta}{R}\right)^{2}\left(1, \delta, \delta^{2}\right) \mathrm{d} \delta
$$

Then, the boundary potential energy for the uniform variable thickness FG spherical torus can be expressed as

$$
\begin{aligned}
U_{b}= & \frac{1}{2} \int_{0}^{2 \pi} \int_{-h / 2}^{h / 2}\left\{k_{u, 0} u^{2}+k_{v, 0} v^{2}+k_{w, 0} w^{2}+k_{\theta, 0} \psi_{\theta}^{2}+k_{\varphi, 0} \psi_{\varphi}^{2}\right\}_{x=x_{l, 0}} \\
& \cdot B \mathrm{~d} \varphi \mathrm{d} \delta+\frac{1}{2} \int_{0}^{2 \pi} \int_{-h / 2}^{h / 2} \\
& \left\{k_{u, 1} u^{2}+k_{v, 1} v^{2}+k_{w, 1} w^{2}+k_{\theta, 1} \psi_{\theta}^{2}+k_{\varphi, 1} \psi_{\varphi}^{2}\right\}_{x=x_{l, 1}} B \mathrm{~d} \varphi \mathrm{d} \delta,
\end{aligned}
$$

where $k_{t, 0}(t=u, v, w, \theta, \varphi)$ and $k_{t, 1}$, respectively, represent the spring stiffness value at both ends of the uniform variable thickness FG spherical torus.

In the case of two adjacent shell segments for the structure, the potential energy stored in springs $\left(k_{u}, k_{v}, k_{w}\right.$, $\left.k_{\theta}, k_{\varphi}\right)$ can be written as

$$
\begin{aligned}
U_{s}^{i}= & \frac{1}{2} \int_{0}^{2 \pi} \int_{-h / 2}^{h / 2}\left\{k_{u}\left(u^{i}-u^{i+1}\right)^{2}+k_{v}\left(v^{i}-v^{i+1}\right)^{2}\right. \\
& \left.+k_{w}\left(w^{i}-w^{i+1}\right)^{2}+k_{\theta}\left(\psi_{\theta}^{i}-\psi_{\theta}^{i+1}\right)^{2}+k_{\varphi}\left(\psi_{\varphi}^{i}-\psi_{\varphi}^{i+1}\right)^{2}\right\}_{i, i+1}
\end{aligned}
$$

$\cdot B \mathrm{~d} \varphi \mathrm{d} \delta$.

Therefore, the total potential energy is shown as below:

$$
U_{B S}=U_{b}+\sum_{i=1}^{H-1} U_{s}^{i}
$$

2.3. Admissible Displacement Fields. In this paper, the authors aim to improve the selection of displacement admissible functions on the basic of domain decomposition method. Thus, the authors try to introduce the unified Jacobi polynomials [47], the value of which are defined in the range of $\Theta \in[-1,1]$. The Recurrence formulas of Jacobi polynomials can be written as 


$$
\begin{gathered}
P_{0}^{(\alpha, \beta)}(\Theta)=1, \\
P_{1}^{(\alpha, \beta)}(\Theta)=\frac{\alpha+\beta+2}{2} \Theta-\frac{\alpha-\beta}{2}, \\
P_{i}^{(\alpha, \beta)}(\Theta) \\
=\frac{(\alpha+\beta+2 i-1)\left\{\alpha^{2}-\beta^{2}+\phi(\alpha+\beta+2 i)(\alpha+\beta+2 i-2)\right\}}{2 i(\alpha+\beta+i)(\alpha+\beta+2 i-2)} \\
\cdot P_{i-1}^{(\alpha, \beta)}(\Theta) \\
-\frac{(\alpha+i-1)(\beta+i-1)(\alpha+\beta+2 i)}{i(\alpha+\beta+i)(\alpha+\beta+2 i-2)} P_{i-2}^{(\alpha, \beta)}(\Theta),
\end{gathered}
$$

where $\alpha, \beta>-1$ and $i=2,3, \ldots$.

The displacement functions based on Jacobi polynomials can be generalized as

$$
\begin{gathered}
u=\sum_{m=0}^{M} U_{m} P_{m}^{(\alpha, \beta)}(\theta) \cos (n \varphi) e^{i \omega t}, \\
v=\sum_{m=0}^{M} V_{m} P_{m}^{(\alpha, \beta)}(\theta) \sin (n \varphi) e^{i \omega t}, \\
w=\sum_{m=0}^{M} W_{m} P_{m}^{(\alpha, \beta)}(\theta) \cos (n \varphi) e^{i \omega t}, \\
\psi_{x}=\sum_{m=0}^{M} \psi_{x m} P_{m}^{(\alpha, \beta)}(\theta) \cos (n \varphi) e^{i \omega t}, \\
\psi_{\theta}=\sum_{m=0}^{M} \psi_{\theta m} P_{m}^{(\alpha, \beta)}(\theta) \cos (n \varphi) e^{i \omega t},
\end{gathered}
$$

where $U_{m}, V_{m}, W_{m}, \psi_{\theta m}$, and $\psi_{\varphi m}$ are unknown coefficients; $n$ and $m$, respectively, represent the semiwave number in circumferential and axial direction. $M$ is highest degrees of $m$.

2.4. Solution Procedure. The ultimate Lagrangian energy functions $\mathscr{L}$ can be shown as

$$
\mathscr{L}=\sum_{i=1}^{H}\left(T^{i}-U^{i}\right)-U_{B S} .
$$

Differentiate the unknown coefficients of equation (20), and the formula can be written as

$$
\frac{\partial \mathscr{L}}{\partial \vartheta}=0, \quad \vartheta=U_{m}, V_{m}, W_{m}, \psi_{\theta m}, \psi_{\varphi m} .
$$

Substituting the formulas mentioned above into equation (21), equation (22) can be obtained:

$$
\left(\mathbf{K}-\omega^{2} \mathbf{M}\right) \mathbf{Q}=0
$$

where $\mathbf{K}$ and $\mathbf{M}$, respectively, represent stiffness matrix and mass matrix, and the symbol $\mathbf{Q}$ is the matrix of unknown coefficients. The frequencies of uniform variable thickness FG spherical torus can be obtained by solving equation (22).

\section{Numerical Results and Discussion}

In this study, the various edge restraints are represented by the first letter of a word. For example, the free and clamped elastic edge conditions can be represented by the letter $\mathrm{F}$ and $\mathrm{C}$. The material properties and geometrical dimensions are chosen as $E_{1}=168 \mathrm{GPa}, E_{2}=70 \mathrm{GPa}$, $\rho_{1}=5700 \mathrm{~kg} / M^{3}, \rho_{2}=2707 \mathrm{~kg} / M^{3}, \mu_{1}=\mu_{2}=0.3, R=1 \mathrm{~m}$, $\theta_{0}=\pi / 4, \theta_{1}=\pi / 2, h_{0}=0.05 \mathrm{~m}$. In addition, other related parameters used in this paper are defined as $M=8, \alpha=0$, $\beta=-0.5, \zeta=4$. The nondimensional frequency is expressed as $\Omega=\omega R \sqrt{\rho_{1}\left(1-\mu_{1}^{2}\right) / E_{1}}$.

3.1. Convergence Analysis. The convergence study of the current method should be carried out before analyzing the free vibration behaviors of FG spherical torus with uniform variable thickness, and the effects of boundary springs, segment numbers, etc. have been reported in this section.

Figure 3 shows the results of FG spherical torus with uniform variable thickness under different boundary parameters. It can be easy to ensure a stable convergence when the stiffness values of connective springs and boundary springs in the range of $10^{3} E_{1} \sim 10^{10} E_{1}$. That is to say, the edge condition is clamped when spring stiffness values are set in this interval. In addition, it should be noted that the edge condition is free when spring stiffness values are less than $10^{-7} E_{1}$. It means that the edge restraints changing from free to clamped case can be simulated with the spring stiffness values within the range of $10^{-11} E_{1} \sim 10^{10} E_{1}$. The corresponding spring stiffness values of general edge conditions used in this paper are displayed in Table 1.

Figure 4 exhibits the convergence study of frequency parameter $\Omega$ for different segment numbers $\zeta$ in $F G$ spherical torus with uniform variable thickness. It is clear that the proposed method converges quickly with the increase of $\zeta$. It can be easy to obtain the great convergence when the segment numbers no less than 3 . In other words, it is not necessary to choose a high value of the segment numbers $\zeta$. Considering the solution precision of FG spherical torus with uniform variable thickness, the value of $\zeta$ is set as 4 in this study.

The relative percentage error of frequency parameter $\Omega$ with different axial wave number about various Jacobi parameters in FG spherical torus is shown in Figure 5, and the results of frequency parameter $\alpha=\beta=1$ are set as the comparative example. It is clear that the different Jacobi parameters have little effect on vibration behaviors of FG spherical torus with uniform variable thickness and the maximum percentage error does not exceed 0.02 . That is to say all the Jacobi polynomials have huge advantages in constructing displacement functions.

Figure 6 illustrates the frequency parameter $\Omega$ with different truncation numbers in FG spherical torus with uniform variable thickness. The results of the first three meridional modes and the lowest four circumferential wave 

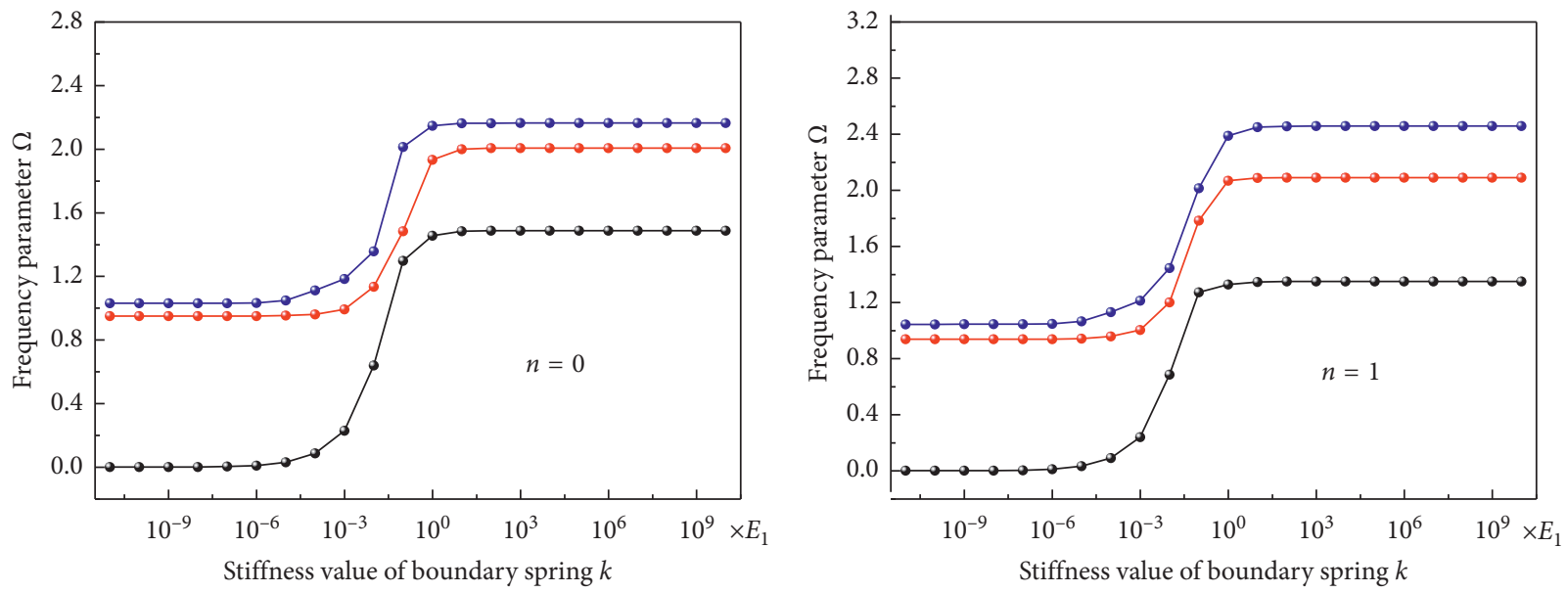

$$
\begin{aligned}
& \multimap m=2 \\
& \multimap m=3 \\
& \multimap m=4
\end{aligned}
$$$$
\begin{aligned}
& \multimap m=2 \\
& \multimap m=3 \\
& \multimap m=4
\end{aligned}
$$

(a)
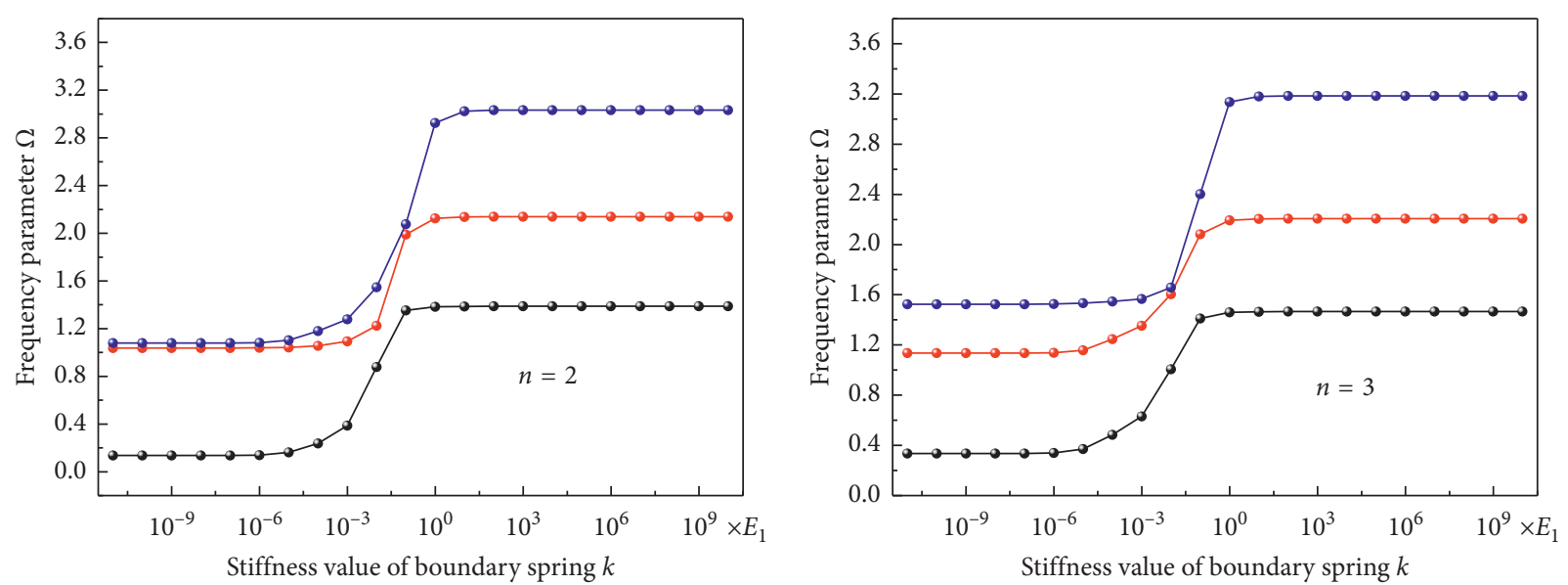

$\rightarrow m=2$

$\rightarrow m=3$

$\rightarrow m=4$

(c)

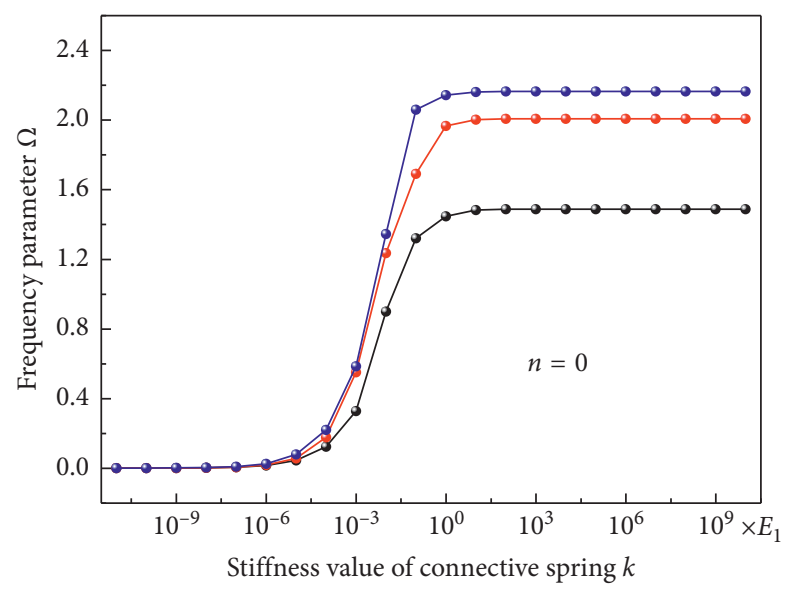

$\because m=2$

$\therefore m=3$

$\longrightarrow m=2$

$\rightarrow m=3$

$\rightarrow m=4$

(d)

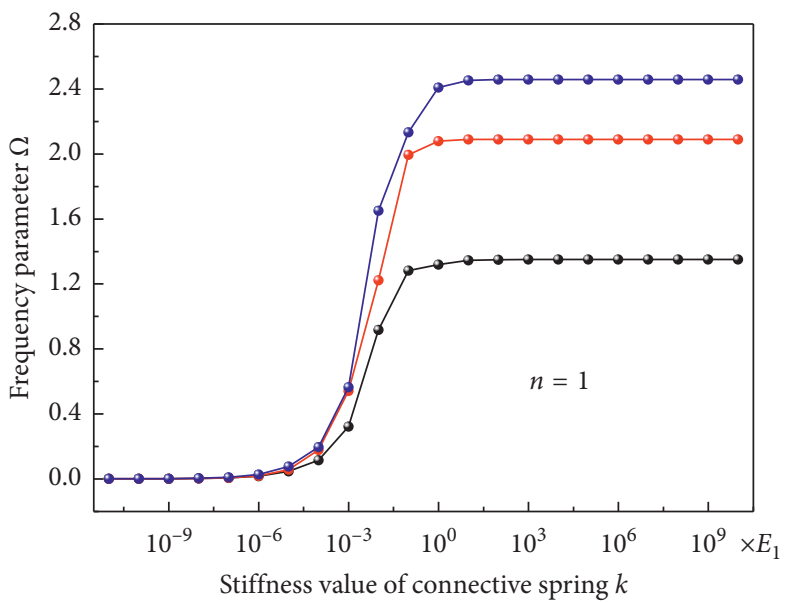

$\rightarrow m=2$

$\rightarrow m=3$

$\rightarrow m=4$

(e)

(f)

Figure 3: Continued. 


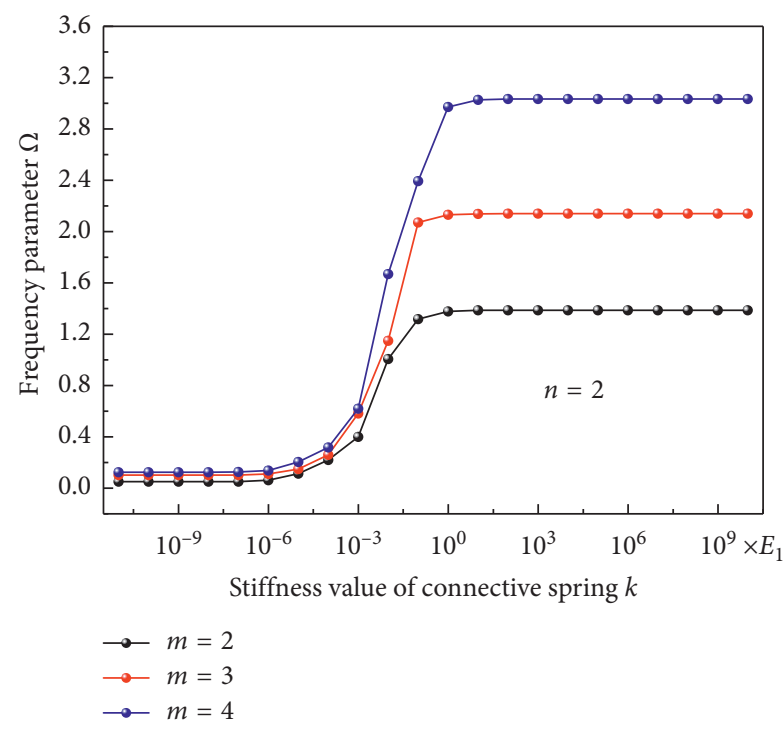

(g)

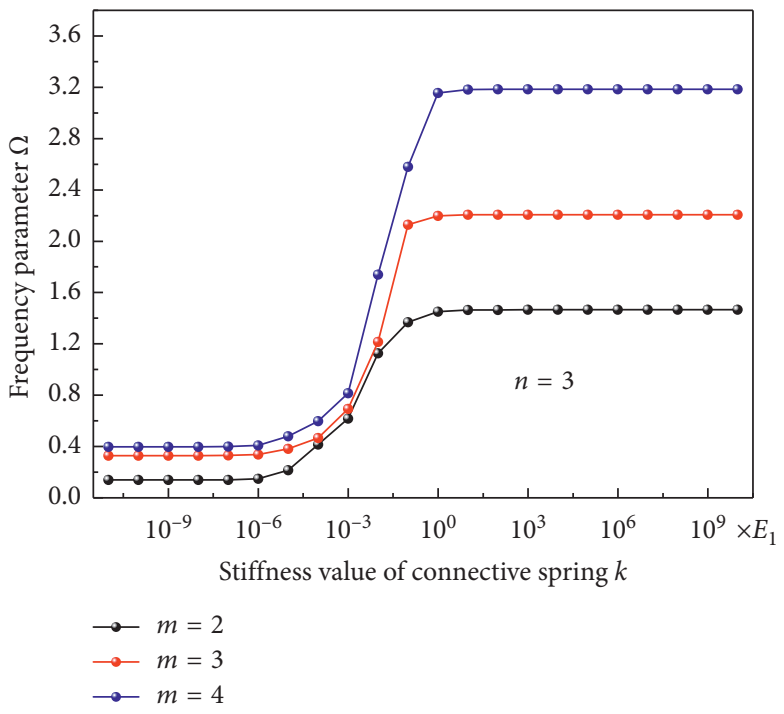

(h)

FIGURE 3: Frequency parameters $\Omega$ of FG spherical shell with uniform variable thickness under different boundary parameters.

TABLE 1: The spring stiffness values of the general edge conditions.

\begin{tabular}{lcccc}
\hline BC & $k_{u, 0}, k_{u, 1}$ & $k_{v, 0}, k_{v, 1}$ & $k_{w, 0}, k_{w, 1}$ & $k_{\theta, 0}, k_{\theta, 1}$ \\
\hline F & 0 & 0 & 0 & 0 \\
SD & 0 & $10^{3} E_{1}$ & $10^{3} E_{1}$ & 0 \\
SS & $10^{3} E_{1}$ & $10^{3} E_{1}$ & $10^{3} E_{1}$ & 0 \\
C & $10^{3} E_{1}$ & $10^{3} E_{1}$ & $10^{3} E_{1}$ & 0 \\
E1 & $10^{-3} E_{1}$ & $10^{3} E_{1}$ & $10^{3} E_{1}$ & $10^{3} E_{1}$ \\
E2 & $10^{3} E_{1}$ & $10^{-3} E_{1}$ & $10^{3} E_{1}$ & $1 E^{3} E_{1}$ \\
E3 & $10^{-3} E_{1}$ & $10^{-3} E_{1}$ & $10^{3} E_{1}$ & $10^{3} E_{1}$ \\
\hline
\end{tabular}

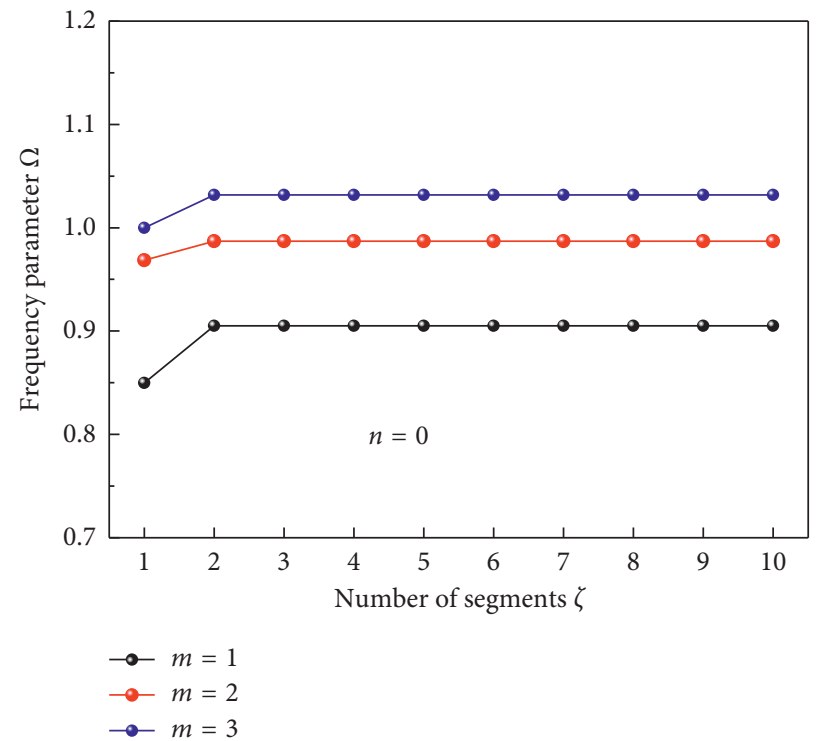

(a)

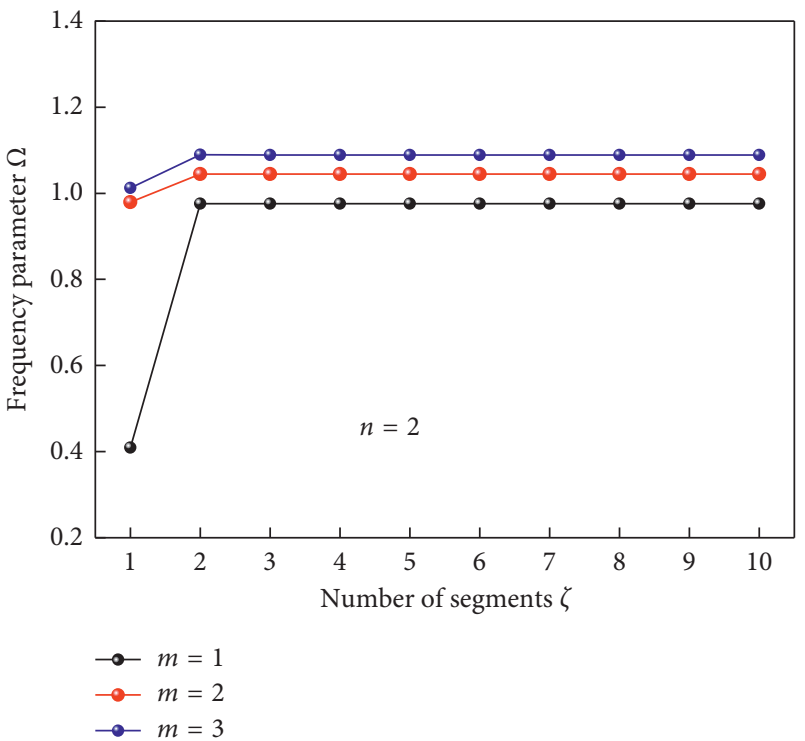

(b)

FIgURe 4: Continued. 


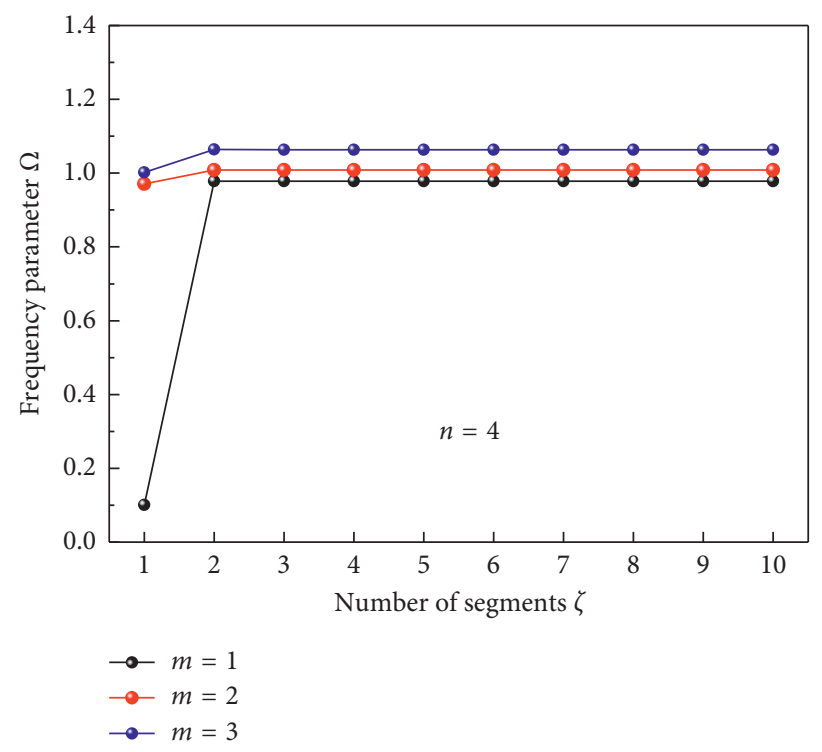

(c)

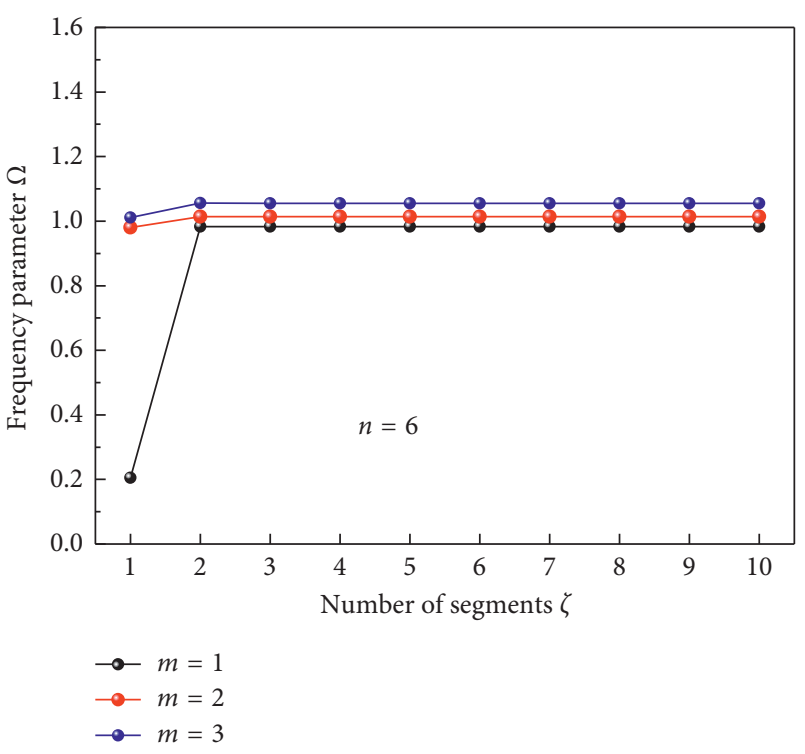

(d)

FIGURE 4: Frequency parameters $\Omega$ of FG spherical shell with uniform variable thickness under different number of segments.

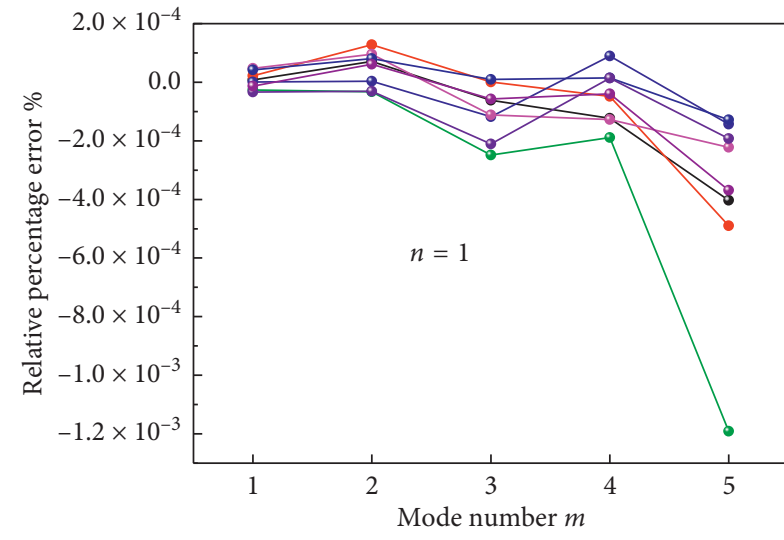

$$
\begin{array}{ll}
\rightarrow \alpha=-0.5, \beta=-0.5 & \rightarrow \alpha=-0.5, \beta=0 \\
\rightarrow \alpha=0, \beta=-0.5 & \rightarrow \alpha=0, \beta=0 \\
\rightarrow \alpha=0.5, \beta=0 & \rightarrow \alpha=0, \beta=0.5 \\
\multimap \alpha=0.5, \beta=0.5 & \rightarrow \alpha=1, \beta=0.5
\end{array}
$$

(a)

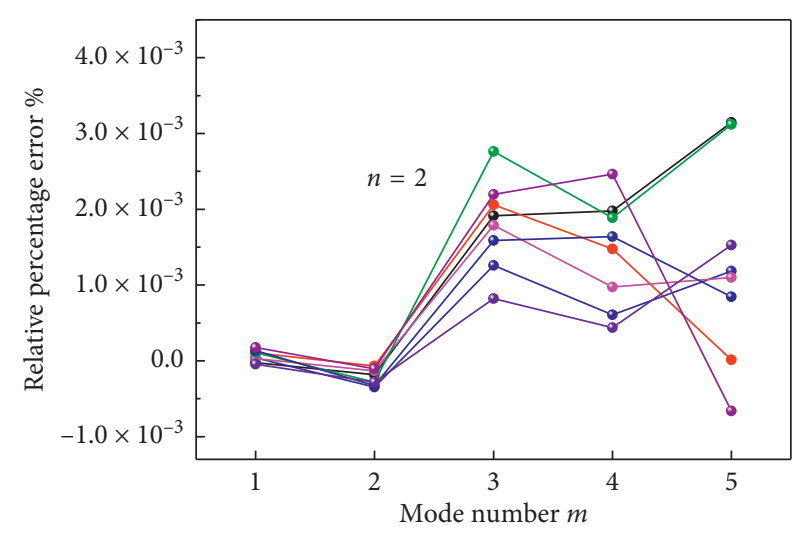

$$
\begin{array}{ll}
\rightarrow \alpha=-0.5, \beta=-0.5 & \rightarrow \alpha=-0.5, \beta=0 \\
\rightarrow \alpha=0, \beta=-0.5 & \rightarrow \alpha=0, \beta=0 \\
\rightarrow \alpha=0.5, \beta=0 & \rightarrow \alpha=0, \beta=0.5 \\
\rightarrow \alpha=0.5, \beta=0.5 & \rightarrow \alpha=1, \beta=0.5
\end{array}
$$

(b)

Figure 5: Continued. 

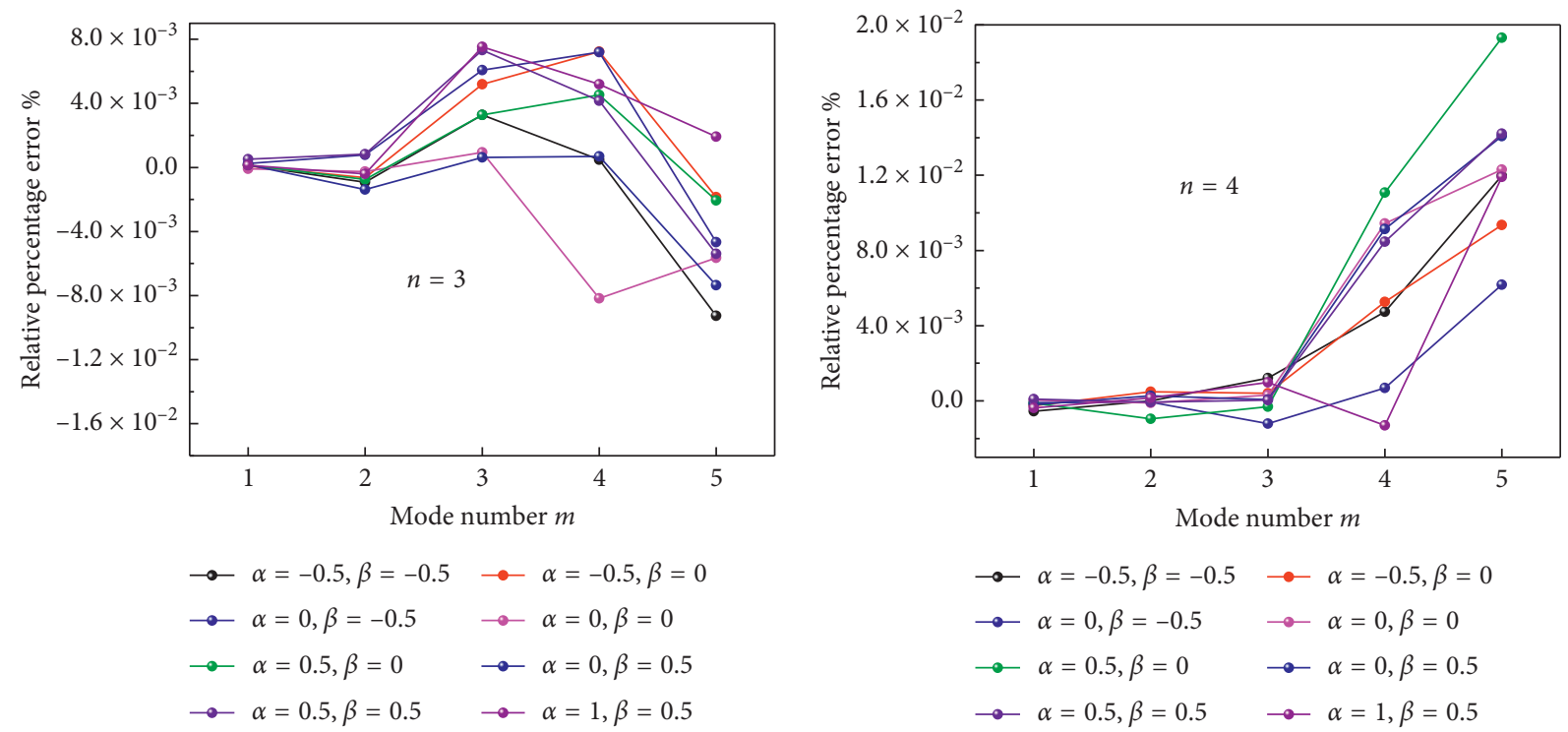

(c)

(d)

FIGURE 5: Relative percentage error of FG spherical shell with uniform variable thickness under different Jacobi parameters.
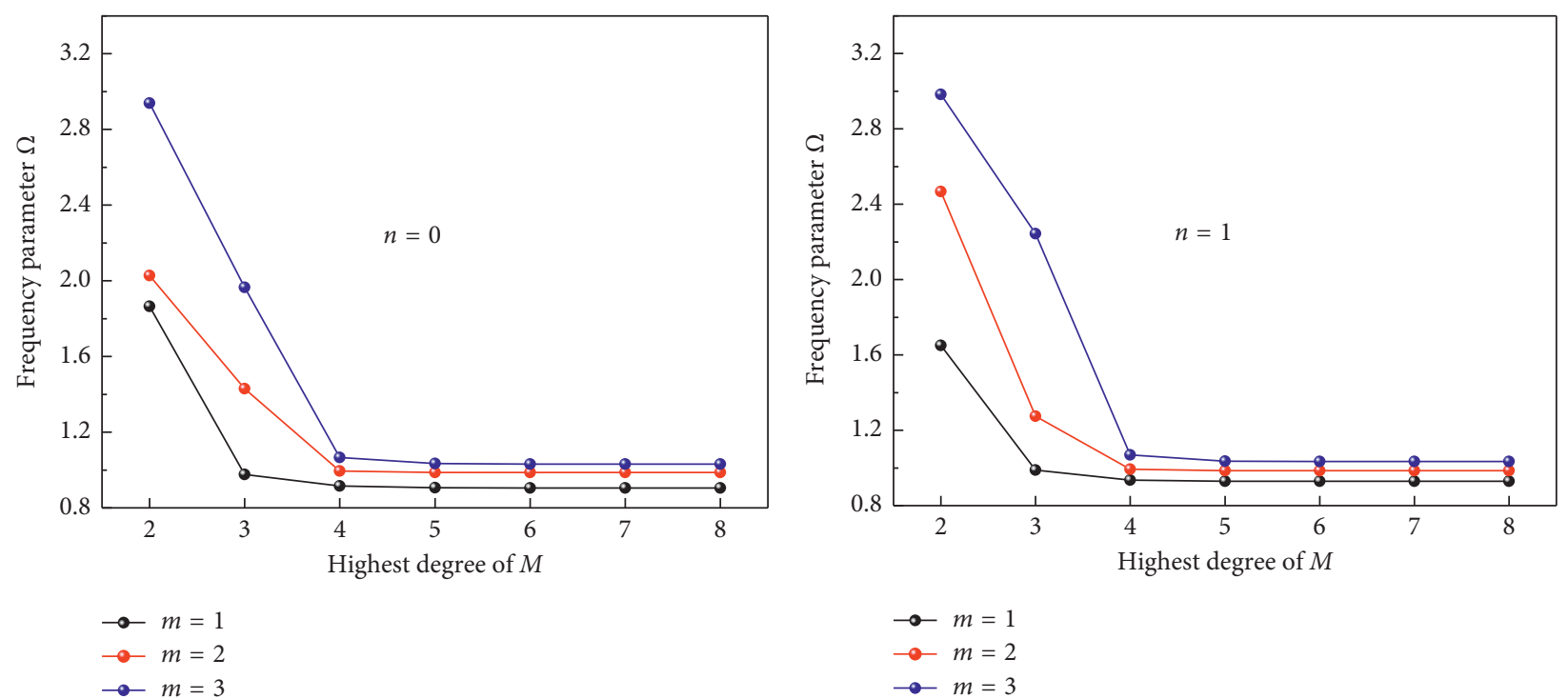

(a)

(b)

Figure 6: Continued. 


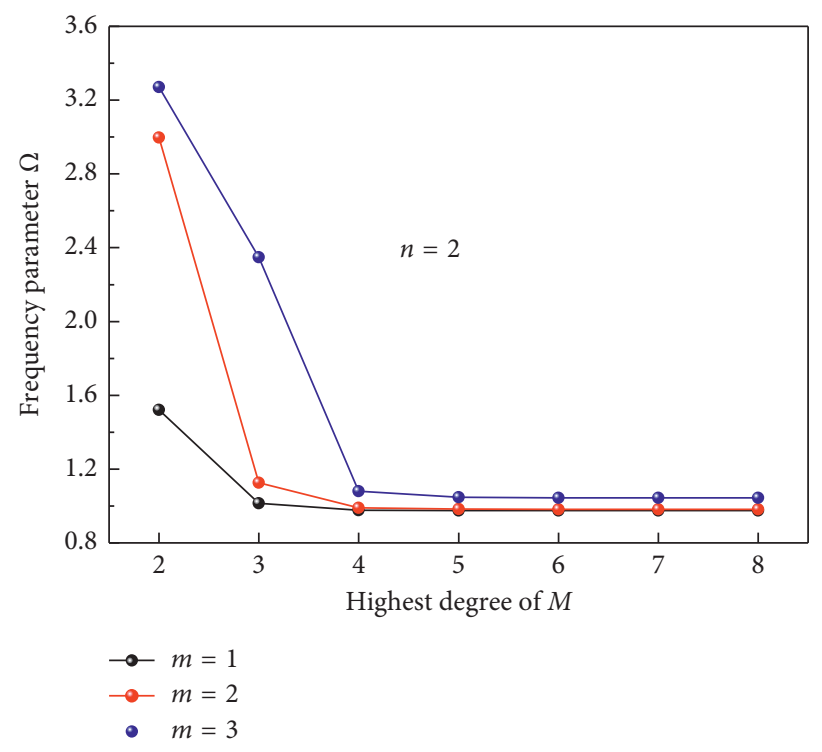

(c)

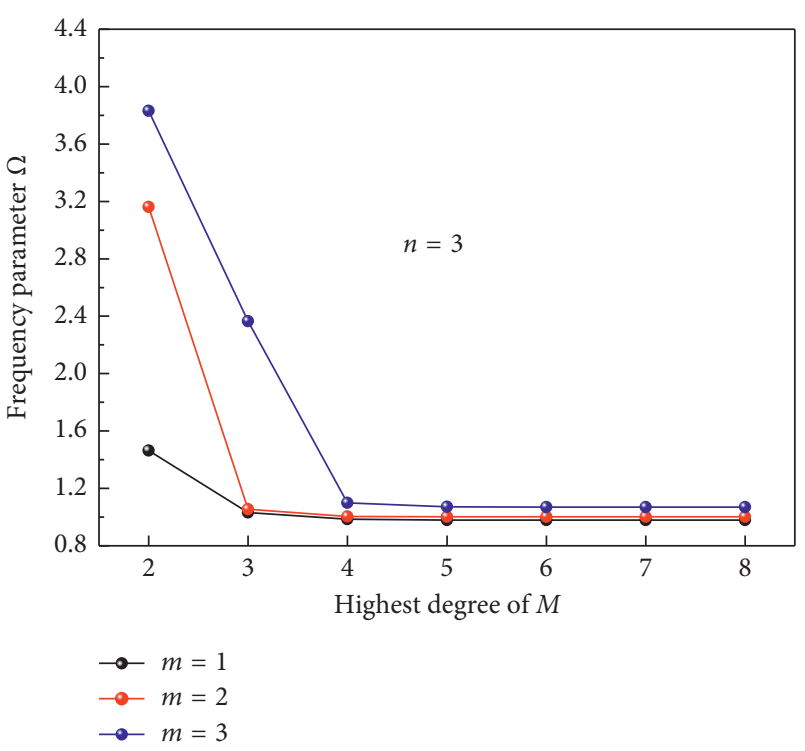

(d)

Figure 6: Frequency parameters $\Omega$ of FG spherical shell with uniform variable thickness under different truncation.

TABLE 2: Comparison of the results for annular isotropic spherical shell.

\begin{tabular}{ccccccccccccc}
\hline$n$ & & F-F & & & F-C & & \multicolumn{3}{c}{ C-F } & & C-C \\
& Present & Ref [21] & FEM & Present & Ref [21] & FEM & Present & Ref [21] & FEM & Present & Ref [21] & FEM \\
\hline 0 & 0.8719 & 0.8705 & 0.8705 & 0.8169 & 0.8166 & 0.8166 & 0.4053 & 0.4055 & 0.4060 & 0.9874 & 0.9871 & 0.9874 \\
1 & 0.8669 & 0.8641 & 0.8643 & 0.6087 & 0.6088 & 0.6089 & 0.1517 & 0.1517 & 0.1518 & 1.0267 & 1.0264 & 1.0268 \\
2 & 0.0572 & 0.0573 & 0.0573 & 0.3485 & 0.3486 & 0.3487 & 0.0763 & 0.0764 & 0.0764 & 1.0032 & 1.0029 & 1.0034 \\
3 & 0.1532 & 0.1532 & 0.1533 & 0.6574 & 0.6573 & 0.6576 & 0.1541 & 0.1541 & 0.1542 & 0.9983 & 0.9980 & 0.9984 \\
4 & 0.2789 & 0.2790 & 0.2792 & 0.9657 & 0.9654 & 0.9659 & 0.2790 & 0.2791 & 0.2793 & 1.0430 & 1.0427 & 1.0431 \\
5 & 0.4293 & 0.4294 & 0.4300 & 1.1159 & 1.1156 & 1.1163 & 0.4293 & 0.4294 & 0.4300 & 1.1266 & 1.1262 & 1.1269 \\
6 & 0.6018 & 0.6019 & 0.6032 & 1.2444 & 1.2440 & 1.2452 & 0.6018 & 0.6019 & 0.6032 & 1.2462 & 1.2458 & 1.2470 \\
7 & 0.7955 & 0.7956 & 0.7981 & 1.4007 & 1.4003 & 1.4025 & 0.7955 & 0.7956 & 0.7981 & 1.4010 & 1.4006 & 1.4028 \\
8 & 1.0104 & 1.0105 & 1.0148 & 1.5899 & 1.5893 & 1.5933 & 1.0104 & 1.0105 & 1.0148 & 1.5899 & 1.5894 & 1.5934 \\
9 & 1.2467 & 1.2467 & 1.2537 & 1.8109 & 1.8103 & 1.8169 & 1.2467 & 1.2467 & 1.2537 & 1.8109 & 1.8103 & 1.8169 \\
\hline
\end{tabular}

numbers $(n=0,1,2,3)$ are considered. The result shows apparently that the current method converges quickly with the value of $M$ increasing and stable convergence can be obtained when the value of $M$ is more than 5 . That is to say, the high value of $M$ is not requisite in admissible displacement functions. In this study, the value of $M$ is set as 8 to guarantee the stable convergence of FG spherical torus with uniform variable thickness.

3.2. Vibration Behavior Analysis of FG Spherical Torus Structure. Table 2 displays the results for uniform isotropic spherical shell with classical edge conditions, and all the results are compared with published literatures and FEM. Table 3 and Figure 7 illustrate the frequency for isotropic spherical shell with uniform variable thickness along the axial direction with classical edge conditions. The results are compared with those obtained by FEM commercial software ABAQUS. From the comparison study, it should be noted that the results of proposed method are highly unified with those obtained by FEM and published literatures. Thus, it can be concluded that the current method is capable to carry out the vibration solutions of uniform isotropic spherical shell and isotropic spherical shell with uniform variable thickness along axial direction.

Table 4 exhibits the natural frequency of FG spherical torus under general boundary conditions, and all the results are compared with those occurred in $\mathrm{Qu}$ [21] and $\mathrm{Su}$ [18]. It can be easy to find that the current method has great accuracy in analyzing the free vibration of FG spherical torus structure from the comparative study; that is to say, the current method is very accurate to solve the free vibration of special form of FG spherical torus with uniform variable thickness.

The results of FG spherical torus with uniform variable thickness subject to classical, elastic, and classical-elastic boundary conditions are shown in Tables 5 and 6 and Figure 8. It is obvious that the frequency parameters have a tendency to rise with the increase of axial modes, while the frequency parameters seems to have no significant change 
TABLE 3: Comparison of frequency for isotropic spherical shell with uniform variable thickness along axial direction.

\begin{tabular}{|c|c|c|c|c|c|c|c|c|c|}
\hline \multirow{2}{*}{$n$} & \multirow{2}{*}{$m$} & \multicolumn{2}{|c|}{$\mathrm{F}-\mathrm{F}$} & \multicolumn{2}{|c|}{$\mathrm{F}-\mathrm{C}$} & \multicolumn{2}{|c|}{$\mathrm{C}-\mathrm{F}$} & \multicolumn{2}{|c|}{$\mathrm{C}-\mathrm{C}$} \\
\hline & & Present & FEM & Present & FEM & Present & FEM & Present & FEM \\
\hline \multirow{4}{*}{2} & 1 & 50.70 & 50.72 & 348.96 & 349.20 & 127.61 & 127.40 & 1036.78 & 1037.50 \\
\hline & 2 & 117.05 & 117.21 & 927.65 & 928.12 & 907.42 & 907.32 & 1207.30 & 1209.00 \\
\hline & 3 & 901.94 & 901.82 & 1244.97 & 1246.40 & 1183.24 & 1185.30 & 1864.31 & 1871.40 \\
\hline & 4 & 939.14 & 939.61 & 1829.09 & 1832.80 & 1449.69 & 1450.90 & 2642.52 & 2653.80 \\
\hline \multirow{4}{*}{4} & 1 & 271.99 & 272.20 & 479.32 & 480.13 & 275.06 & 275.26 & 998.93 & 999.58 \\
\hline & 2 & 486.57 & 487.43 & 1042.06 & 1043.30 & 1012.90 & 1013.80 & 1354.10 & 1356.70 \\
\hline & 3 & 1060.15 & 1061.10 & 1440.20 & 1443.10 & 1388.19 & 1391.00 & 2004.86 & 2012.60 \\
\hline & 4 & 1475.23 & 1478.20 & 2094.70 & 2101.50 & 2030.25 & 2032.80 & 2890.96 & 2912.10 \\
\hline \multirow{4}{*}{6} & 1 & 597.10 & 597.97 & 934.88 & 937.69 & 597.98 & 598.83 & 1140.47 & 1141.60 \\
\hline & 2 & 944.78 & 947.81 & 1244.86 & 1247.40 & 1186.36 & 1188.00 & 1569.72 & 1573.20 \\
\hline & 3 & 1300.91 & 1303.90 & 1725.41 & 1730.30 & 1641.09 & 1645.00 & 2244.57 & 2253.10 \\
\hline & 4 & 1796.29 & 1801.50 & 2410.55 & 2419.70 & 2328.08 & 2337.00 & 3144.42 & 3166.80 \\
\hline
\end{tabular}

FEM

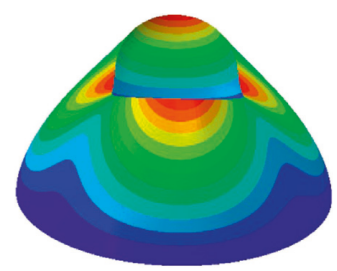

F-C

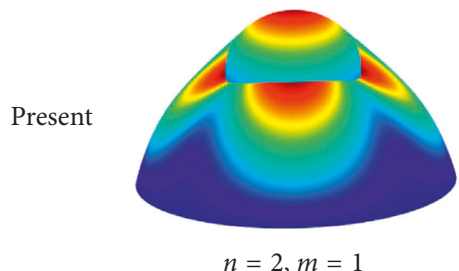

$n=2, m=1$

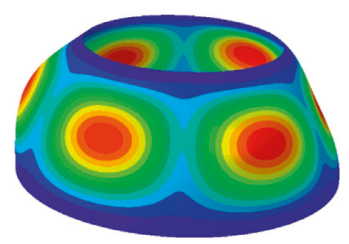

C-C

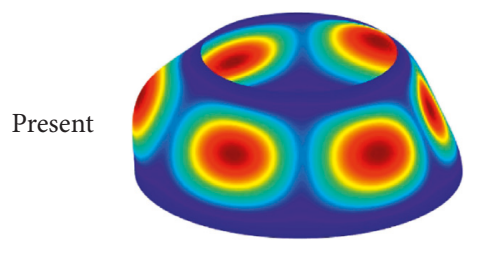

$n=3, m=1$
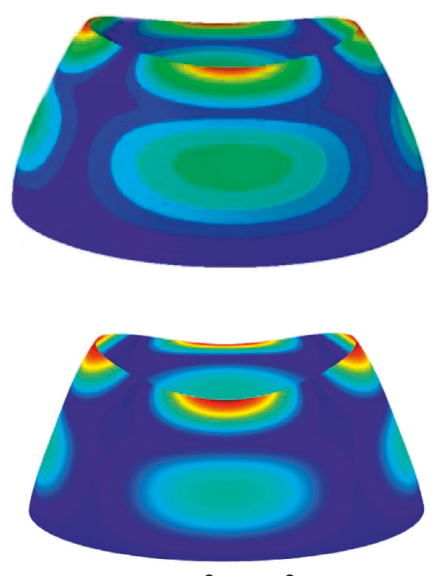

$n=2, m=2$
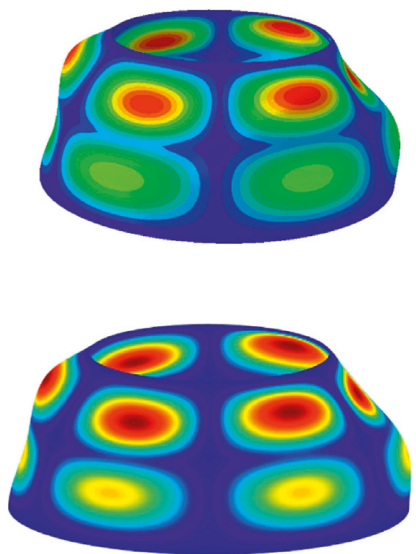

$n=3, m=2$
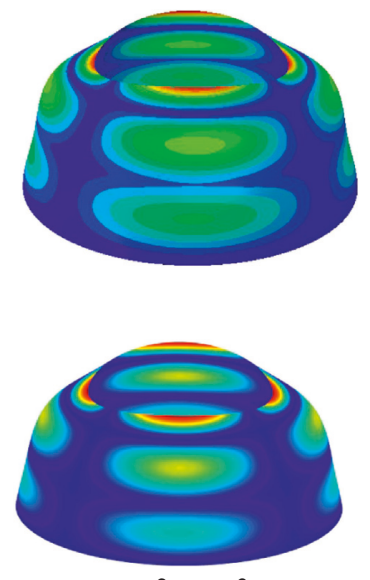

$n=2, m=3$
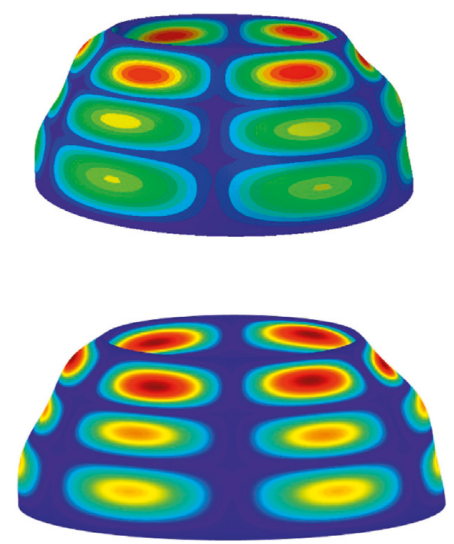

$n=3, m=3$

FIgURE 7: Comparison of the FEM and predicted mode shapes for the spherical shell structure.

with the increase of circumferential modes. Moreover, it is apparent that the results of the structure subject to clamped boundary condition are higher than those subject to other boundary conditions. In other words, the edge restraints have an apparent effect on the vibration behaviors of FG spherical torus with uniform variable thickness from the examples.
Table 7 shows the results of FG spherical torus with uniform variable thickness under different $h_{0} / R$ ratio and axial modes, in which five different kinds of $h_{0} / R$ are included. It can be seen that the results of the structure tend to rise with the increase of $h_{0} / R$ and axial modes when the boundary condition and circumferential mode are fixed. Figure 9 illustrates the frequency parameter curve of FG 
TABLE 4: Comparison of the natural frequency for FG spherical shell $(m=1)$.

\begin{tabular}{lcccccccccc}
\hline \multirow{2}{*}{ Type } & & & \multicolumn{3}{c}{ F-F } & & & SS-SS & & C-C \\
& & $n$ & Present & Ref [21] & Ref [18] & Present & Ref [21] & Ref [18] & Present & Ref [21] \\
Ref [18]
\end{tabular}

TABLE 5: Results of FG spherical shell with uniform variable thickness subject to classical and elastic edge conditions $\left(\theta_{0}=\pi / 4, \theta_{\zeta}=\pi / 2\right.$, $\left.h_{0}=0.05 \mathrm{~m}, R=1 \mathrm{~m}, a=1 / \mathrm{b}=0.5 / c=1 / p=1\right)$.

\begin{tabular}{|c|c|c|c|c|c|c|c|c|c|c|c|}
\hline \multirow{2}{*}{ Type } & \multirow{2}{*}{$n$} & \multirow{2}{*}{$m$} & \multicolumn{9}{|c|}{ Edge conditions } \\
\hline & & & $\mathrm{F}-\mathrm{C}$ & $\mathrm{F}-\mathrm{SD}$ & $\mathrm{F}-\mathrm{SS}$ & $\mathrm{C}-\mathrm{C}$ & $\mathrm{C}-\mathrm{SD}$ & $\mathrm{C}-\mathrm{SS}$ & E1-E1 & E2-E2 & E3-E3 \\
\hline \multirow{18}{*}{$\mathrm{FGM}_{\mathrm{I}}$} & \multirow{6}{*}{1} & 1 & 0.8061 & 0.8515 & 0.7695 & 1.4743 & 0.8666 & 1.4093 & 0.7110 & 0.7212 & 0.2475 \\
\hline & & 2 & 1.0844 & 1.0406 & 1.0226 & 1.7257 & 1.4854 & 1.5449 & 1.1898 & 1.7130 & 0.5718 \\
\hline & & 3 & 1.7518 & 1.5909 & 1.5809 & 2.6423 & 2.0498 & 2.5978 & 1.7672 & 1.8102 & 1.4872 \\
\hline & & 4 & 2.1285 & 1.9103 & 2.1195 & 3.0188 & 2.6236 & 2.7635 & 2.5254 & 2.7277 & 1.7468 \\
\hline & & 5 & 2.4997 & 1.9103 & 2.4987 & 4.0681 & 2.9832 & 3.9842 & 2.9350 & 3.0070 & 2.9186 \\
\hline & & 6 & 3.0641 & 2.1570 & 2.7648 & 4.6160 & 4.1743 & 4.3795 & 4.4855 & 4.2229 & 3.0165 \\
\hline & \multirow{6}{*}{2} & 1 & 0.4738 & 0.1189 & 0.4319 & 1.3466 & 1.0130 & 1.2877 & 1.0765 & 1.0010 & 0.5706 \\
\hline & & 2 & 1.1650 & 1.0189 & 1.0935 & 1.7804 & 1.5538 & 1.5854 & 1.3907 & 1.7687 & 0.7426 \\
\hline & & 3 & 1.8301 & 1.0938 & 1.6483 & 2.9556 & 2.0093 & 2.7002 & 1.8000 & 2.4582 & 1.7623 \\
\hline & & 4 & 2.4691 & 1.6660 & 2.4686 & 3.3752 & 2.6982 & 3.3419 & 2.9086 & 2.9094 & 2.2710 \\
\hline & & 5 & 2.9391 & 2.7779 & 2.7936 & 4.0456 & 3.5972 & 3.9935 & 2.9754 & 3.1337 & 2.9568 \\
\hline & & 6 & 3.1283 & 2.8807 & 2.9793 & 4.6017 & 4.2218 & 4.3309 & 4.5264 & 4.4187 & 3.7186 \\
\hline & \multirow{6}{*}{3} & 1 & 0.3786 & 0.2948 & 0.3498 & 1.2871 & 1.0927 & 1.2245 & 1.1397 & 1.1053 & 0.8530 \\
\hline & & 2 & 1.2615 & 1.1340 & 1.1833 & 1.8613 & 1.6617 & 1.6668 & 1.8575 & 1.8406 & 1.1818 \\
\hline & & 3 & 1.9470 & 1.6207 & 1.7557 & 3.0503 & 2.2682 & 2.7777 & 1.9651 & 2.9568 & 1.8673 \\
\hline & & 4 & 2.6336 & 1.7632 & 2.6335 & 4.0005 & 2.7748 & 3.9978 & 3.0411 & 3.1673 & 2.9612 \\
\hline & & 5 & 3.1661 & 2.8741 & 2.8910 & 4.2552 & 4.1804 & 4.1878 & 3.4069 & 3.7308 & 3.1233 \\
\hline & & 6 & 3.8750 & 3.3357 & 3.8718 & 4.6471 & 4.3587 & 4.3904 & 4.5893 & 4.5535 & 4.2193 \\
\hline \multirow{18}{*}{$\mathrm{FGM}_{\mathrm{II}}$} & \multirow{6}{*}{1} & 1 & 0.8036 & 0.8425 & 0.7625 & 1.4689 & 0.8635 & 1.4241 & 0.7085 & 0.7188 & 0.2468 \\
\hline & & 2 & 1.0797 & 0.9655 & 1.0147 & 1.7168 & 1.4789 & 1.5221 & 1.1857 & 1.7041 & 0.5693 \\
\hline & & 3 & 1.7431 & 0.9655 & 1.5624 & 2.6349 & 2.0434 & 2.5781 & 1.7581 & 1.8044 & 1.4827 \\
\hline & & 4 & 2.1233 & 1.0453 & 2.1184 & 3.0038 & 2.6117 & 2.7764 & 2.5192 & 2.7204 & 1.7377 \\
\hline & & 5 & 2.4916 & 1.6326 & 2.4759 & 4.0550 & 2.9734 & 3.9230 & 2.9195 & 2.9916 & 2.9041 \\
\hline & & 6 & 3.0495 & 2.1457 & 2.7919 & 4.5957 & 4.1517 & 4.4147 & 4.4618 & 4.2061 & 3.0081 \\
\hline & & 1 & 0.4722 & 0.1183 & 0.4224 & 1.3414 & 1.0103 & 1.2975 & 1.0719 & 0.9970 & 0.5690 \\
\hline & \multirow{5}{*}{2} & 2 & 1.1600 & 1.0160 & 1.0894 & 1.7711 & 1.5469 & 1.5699 & 1.3870 & 1.7594 & 0.7390 \\
\hline & & 3 & 1.8209 & 1.0897 & 1.6321 & 2.9406 & 2.0032 & 2.6845 & 1.7906 & 2.4509 & 1.7532 \\
\hline & & 4 & 2.4612 & 1.6581 & 2.4575 & 3.3662 & 2.6846 & 3.3483 & 2.9013 & 2.8995 & 2.2647 \\
\hline & & 5 & 2.9311 & 2.7631 & 2.7934 & 4.0339 & 3.5870 & 3.9432 & 2.9598 & 3.1210 & 2.9416 \\
\hline & & 6 & 3.1140 & 2.8737 & 2.9857 & 4.5796 & 4.2000 & 4.3519 & 4.5027 & 4.3972 & 3.7091 \\
\hline & \multirow{6}{*}{3} & 1 & 0.3767 & 0.2933 & 0.3434 & 1.2819 & 1.0908 & 1.2318 & 1.1348 & 1.1006 & 0.8490 \\
\hline & & 2 & 1.2561 & 1.1314 & 1.1845 & 1.8516 & 1.6538 & 1.6569 & 1.8477 & 1.8308 & 1.1780 \\
\hline & & 3 & 1.9371 & 1.6153 & 1.7440 & 3.0338 & 2.2623 & 2.7635 & 1.9599 & 2.9425 & 1.8581 \\
\hline & & 4 & 2.6255 & 1.7556 & 2.6188 & 3.9898 & 2.7606 & 3.9633 & 3.0247 & 3.1576 & 2.9462 \\
\hline & & 5 & 3.1501 & 2.8592 & 2.8917 & 4.2447 & 4.1618 & 4.1886 & 3.3985 & 3.7214 & 3.1145 \\
\hline & & 6 & 3.8656 & 3.3272 & 3.8656 & 4.6241 & 4.3439 & 4.3944 & 4.5654 & 4.5302 & 4.2081 \\
\hline
\end{tabular}


TABLE 6: Results of FG spherical shell with uniform variable thickness subject to classical-elastic edge conditions $\left(\theta_{0}=\pi / 4, \theta_{\zeta}=\pi / 2\right.$, $\left.h_{0}=0.05 \mathrm{~m}, R=1 \mathrm{~m}, a=1 / \mathrm{b}=0.5 / c=1 / p=1\right)$

\begin{tabular}{|c|c|c|c|c|c|c|c|c|c|c|c|}
\hline \multirow{2}{*}{ Type } & \multirow{2}{*}{$n$} & \multirow{2}{*}{$m$} & \multicolumn{9}{|c|}{ Edge conditions } \\
\hline & & & F-E1 & F-E2 & F-E3 & $\mathrm{C}-\mathrm{E} 1$ & $\mathrm{C}-\mathrm{E} 2$ & C-E3 & SD-E1 & SD-E2 & SD-E3 \\
\hline \multirow{18}{*}{$\mathrm{FGM}_{\mathrm{I}}$} & \multirow{6}{*}{1} & 1 & 0.1745 & 0.4219 & 0.1731 & 0.9155 & 1.0662 & 0.6921 & 0.6555 & 0.9871 & 0.3861 \\
\hline & & 2 & 0.8606 & 1.0758 & 0.4417 & 1.7104 & 1.7134 & 1.4281 & 1.1713 & 1.4578 & 1.1026 \\
\hline & & 3 & 1.1189 & 1.4545 & 1.0907 & 2.0695 & 1.8432 & 1.7637 & 1.5714 & 1.5141 & 1.4661 \\
\hline & & 4 & 1.8136 & 1.7704 & 1.4641 & 2.7920 & 3.0021 & 2.2959 & 2.5230 & 2.5401 & 1.6211 \\
\hline & & 5 & 2.1632 & 2.3807 & 1.8299 & 3.1121 & 3.5931 & 3.0201 & 2.6604 & 2.8782 & 2.6603 \\
\hline & & 6 & 3.0061 & 2.9868 & 2.9843 & 4.4865 & 4.2580 & 3.8824 & 4.1531 & 3.7194 & 3.6927 \\
\hline & \multirow{6}{*}{2} & 1 & 0.1933 & 0.3800 & 0.1874 & 1.0767 & 1.1380 & 0.7658 & 1.0242 & 1.0231 & 0.6860 \\
\hline & & 2 & 1.0412 & 1.1309 & 0.6800 & 1.7613 & 1.7723 & 1.7434 & 1.3829 & 1.5690 & 1.3580 \\
\hline & & 3 & 1.1660 & 1.8245 & 1.1514 & 2.0304 & 2.4699 & 2.0269 & 1.6101 & 2.0579 & 1.5706 \\
\hline & & 4 & 1.8698 & 1.9600 & 1.8267 & 2.9668 & 3.0760 & 2.4819 & 2.7003 & 2.6571 & 2.2832 \\
\hline & & 5 & 2.8811 & 2.7052 & 2.2975 & 3.6263 & 3.6515 & 3.0777 & 2.9070 & 3.2501 & 2.7146 \\
\hline & & 6 & 3.0606 & 3.1279 & 3.0580 & 4.5289 & 4.4547 & 4.4066 & 4.1992 & 3.9565 & 3.8826 \\
\hline & \multirow{6}{*}{3} & 1 & 0.3178 & 0.3600 & 0.3176 & 1.1726 & 1.1774 & 0.9256 & 1.0892 & 1.0714 & 0.8863 \\
\hline & & 2 & 1.2268 & 1.2148 & 0.9903 & 1.8612 & 1.8500 & 1.8494 & 1.6712 & 1.6529 & 1.6088 \\
\hline & & 3 & 1.6334 & 1.9387 & 1.4638 & 2.2805 & 2.9585 & 2.1829 & 1.9613 & 2.6623 & 1.8107 \\
\hline & & 4 & 1.9615 & 2.3374 & 1.9387 & 3.0505 & 3.3536 & 2.9825 & 2.7730 & 2.7692 & 2.7441 \\
\hline & & 5 & 3.1516 & 3.1079 & 3.0559 & 4.2551 & 4.0116 & 3.4333 & 3.4013 & 3.9011 & 3.1271 \\
\hline & & 6 & 3.3389 & 3.6991 & 3.1786 & 4.6062 & 4.5850 & 4.5660 & 4.2698 & 4.2883 & 4.1731 \\
\hline \multirow{18}{*}{$\mathrm{FGM}_{\text {II }}$} & \multirow{6}{*}{1} & 1 & 0.1740 & 0.4204 & 0.1727 & 0.9116 & 1.0626 & 0.6890 & 0.6528 & 0.9840 & 0.3845 \\
\hline & & 2 & 0.8576 & 1.0713 & 0.4400 & 1.7025 & 1.7044 & 1.4237 & 1.1678 & 1.4534 & 1.0992 \\
\hline & & 3 & 1.1141 & 1.4503 & 1.0862 & 2.0630 & 1.8375 & 1.7553 & 1.5638 & 1.5068 & 1.4605 \\
\hline & & 4 & 1.8042 & 1.7622 & 1.4595 & 2.7821 & 2.9863 & 2.2892 & 2.5167 & 2.5292 & 1.6146 \\
\hline & & 5 & 2.1584 & 2.3732 & 1.8209 & 3.0990 & 3.5838 & 3.0043 & 2.6461 & 2.8676 & 2.6461 \\
\hline & & 6 & 2.9898 & 2.9728 & 2.9719 & 4.4628 & 4.2411 & 3.8726 & 4.1306 & 3.7097 & 3.6832 \\
\hline & & 1 & 0.1924 & 0.3784 & 0.1866 & 1.0721 & 1.1336 & 0.7622 & 1.0206 & 1.0205 & 0.6831 \\
\hline & \multirow{5}{*}{2} & 2 & 1.0374 & 1.1261 & 0.6772 & 1.7532 & 1.7630 & 1.7353 & 1.3788 & 1.5614 & 1.3548 \\
\hline & & 3 & 1.1610 & 1.8151 & 1.1466 & 2.0238 & 2.4627 & 2.0202 & 1.6032 & 2.0527 & 1.5632 \\
\hline & & 4 & 1.8601 & 1.9550 & 1.8172 & 2.9514 & 3.0605 & 2.4748 & 2.6864 & 2.6447 & 2.2769 \\
\hline & & 5 & 2.8744 & 2.6961 & 2.2915 & 3.6172 & 3.6422 & 3.0621 & 2.8999 & 3.2396 & 2.7009 \\
\hline & & 6 & 3.0440 & 3.1135 & 3.0414 & 4.5051 & 4.4333 & 4.3916 & 4.1767 & 3.9465 & 3.8722 \\
\hline & \multirow{6}{*}{3} & 1 & 0.3161 & 0.3581 & 0.3159 & 1.1675 & 1.1726 & 0.9212 & 1.0865 & 1.0692 & 0.8831 \\
\hline & & 2 & 1.2214 & 1.2096 & 0.9859 & 1.8514 & 1.8402 & 1.8395 & 1.6639 & 1.6456 & 1.6044 \\
\hline & & 3 & 1.6284 & 1.9287 & 1.4588 & 2.2741 & 2.9447 & 2.1770 & 1.9559 & 2.6526 & 1.8037 \\
\hline & & 4 & 1.9512 & 2.3310 & 1.9287 & 3.0340 & 3.3427 & 2.9677 & 2.7593 & 2.7590 & 2.7310 \\
\hline & & 5 & 3.1345 & 3.0914 & 3.0451 & 4.2446 & 4.0010 & 3.4229 & 3.3930 & 3.8867 & 3.1192 \\
\hline & & 6 & 3.3305 & 3.6897 & 3.1643 & 4.5823 & 4.5624 & 4.5424 & 4.2476 & 4.2748 & 4.1545 \\
\hline
\end{tabular}

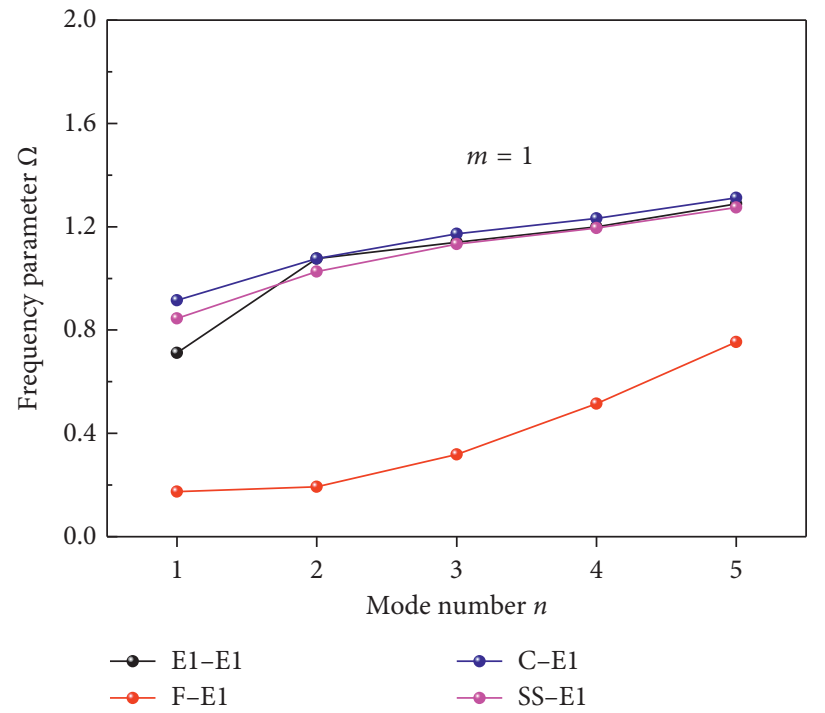

(a)

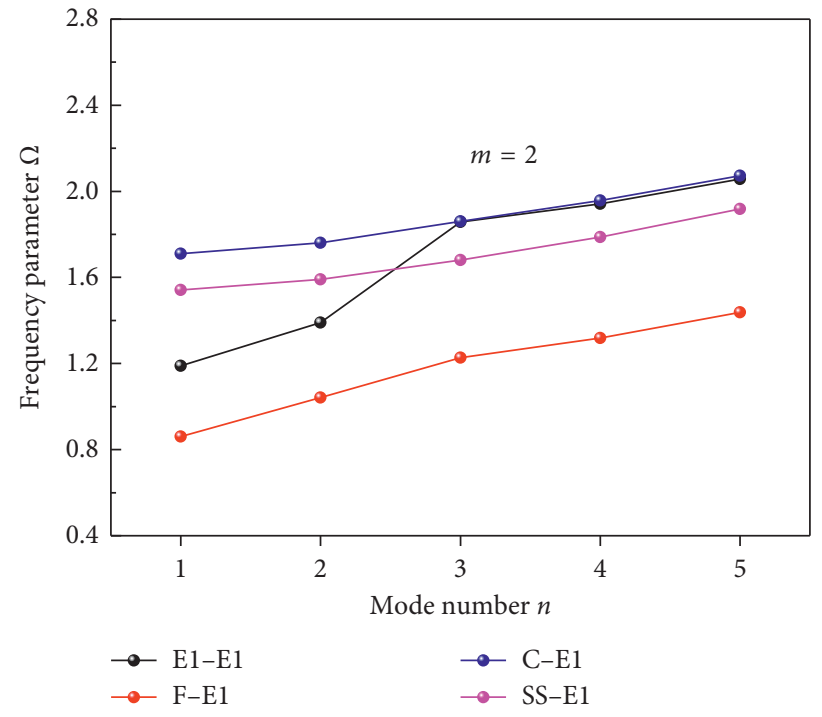

(b)

FIgure 8: Continued. 


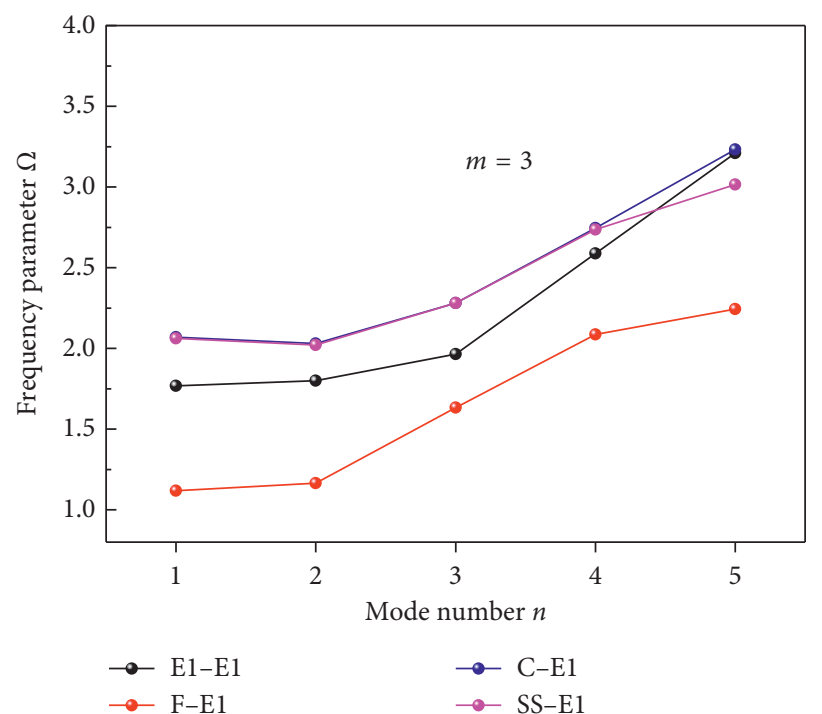

(c)

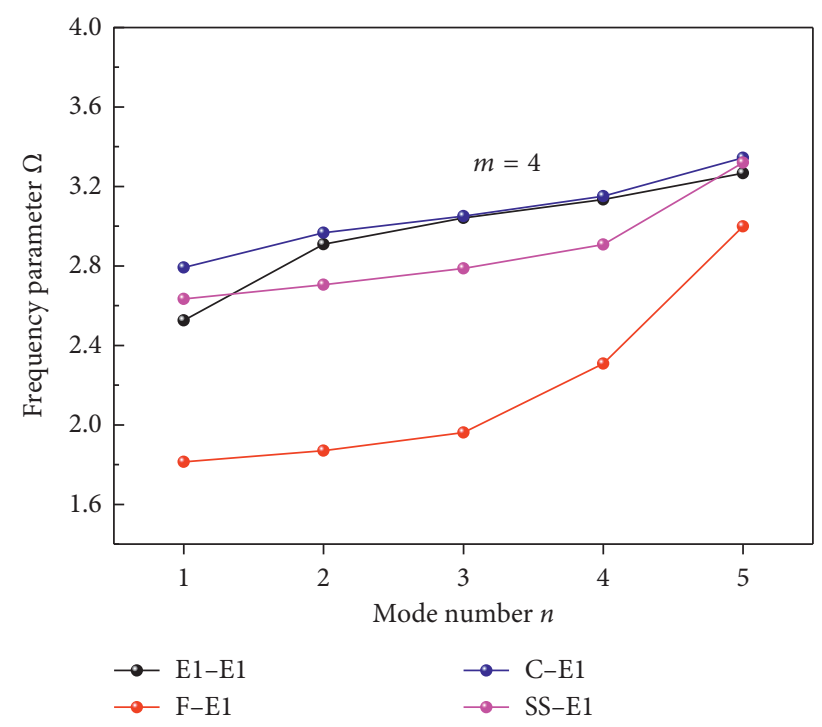

(d)

FIGURE 8: Frequency parameters $\Omega$ of FG spherical shell with uniform variable thickness under different boundary conditions.

TABLE 7: Results of FG spherical shell with uniform variable thickness under different $h_{0} / \mathrm{R}$ ratio $\left(\theta_{0}=\pi / 4, \theta_{\zeta}=\pi / 2, n=2, a=1 / \mathrm{b}=0.5 /\right.$ $c=1 / p=1)$.

\begin{tabular}{|c|c|c|c|c|c|c|c|}
\hline \multirow{2}{*}{ Type } & \multirow{2}{*}{ Edge conditions } & \multirow{2}{*}{$h_{0} / R$} & \multicolumn{5}{|c|}{$m$} \\
\hline & & & 1 & 2 & 3 & 4 & 5 \\
\hline \multirow{20}{*}{$\mathrm{FGM}_{\mathrm{I}}$} & \multirow{5}{*}{$\mathrm{F}-\mathrm{C}$} & 0.03 & 0.4432 & 1.0481 & 1.3822 & 2.0679 & 2.4996 \\
\hline & & 0.05 & 0.4738 & 1.1650 & 1.8301 & 2.4691 & 2.9391 \\
\hline & & 0.07 & 0.5088 & 1.3057 & 2.2010 & 2.5746 & 2.9715 \\
\hline & & 0.09 & 0.5477 & 1.4510 & 2.3658 & 2.7963 & 3.0043 \\
\hline & & 0.11 & 0.5889 & 1.5890 & 2.4201 & 2.9234 & 3.1775 \\
\hline & \multirow{5}{*}{$\mathrm{C}-\mathrm{C}$} & 0.03 & 1.2589 & 1.3556 & 2.1041 & 3.0770 & 3.3356 \\
\hline & & 0.05 & 1.3466 & 1.7804 & 2.9556 & 3.3752 & 4.0456 \\
\hline & & 0.07 & 1.4468 & 2.1796 & 3.2748 & 3.7918 & 4.1087 \\
\hline & & 0.09 & 1.5525 & 2.5145 & 3.3055 & 4.1287 & 4.3264 \\
\hline & & 0.11 & 1.6557 & 2.7807 & 3.3195 & 4.1461 & 4.7406 \\
\hline & \multirow{5}{*}{$\mathrm{C}-\mathrm{SD}$} & 0.03 & 0.9675 & 1.2376 & 1.8193 & 2.0536 & 2.8547 \\
\hline & & 0.05 & 1.0130 & 1.5538 & 2.0093 & 2.6982 & 3.5972 \\
\hline & & 0.07 & 1.0726 & 1.8177 & 2.1034 & 3.3445 & 3.6618 \\
\hline & & 0.09 & 1.1398 & 1.9186 & 2.3257 & 3.5585 & 4.0219 \\
\hline & & 0.11 & 1.2093 & 1.9482 & 2.5766 & 3.5856 & 4.4516 \\
\hline & \multirow{5}{*}{ C-E1 } & 0.03 & 1.0025 & 1.3522 & 1.9276 & 2.1570 & 3.1102 \\
\hline & & 0.05 & 1.0767 & 1.7613 & 2.0304 & 2.9668 & 3.6263 \\
\hline & & 0.07 & 1.1679 & 1.9748 & 2.2419 & 3.4964 & 3.8330 \\
\hline & & 0.09 & 1.2610 & 2.0140 & 2.5679 & 3.5702 & 4.3233 \\
\hline & & 0.11 & 1.3466 & 2.0409 & 2.8447 & 3.5890 & 4.7302 \\
\hline
\end{tabular}


TABle 7: Continued.

\begin{tabular}{|c|c|c|c|c|c|c|c|}
\hline \multirow{2}{*}{ Type } & \multirow{2}{*}{ Edge conditions } & \multirow{2}{*}{$h_{0} / R$} & \multicolumn{5}{|c|}{$m$} \\
\hline & & & 1 & 2 & 3 & 4 & 5 \\
\hline \multirow{20}{*}{$\mathrm{FGM}_{\mathrm{II}}$} & \multirow{5}{*}{$\mathrm{F}-\mathrm{C}$} & 0.03 & 0.4424 & 1.0457 & 1.3782 & 2.0609 & 2.4954 \\
\hline & & 0.05 & 0.4722 & 1.1600 & 1.8209 & 2.4612 & 2.9311 \\
\hline & & 0.07 & 0.5062 & 1.2973 & 2.1878 & 2.5618 & 2.9619 \\
\hline & & 0.09 & 0.5438 & 1.4387 & 2.3527 & 2.7753 & 2.9904 \\
\hline & & 0.11 & 0.5837 & 1.5724 & 2.4057 & 2.9053 & 3.1498 \\
\hline & \multirow{6}{*}{$\mathrm{C}-\mathrm{C}$} & 0.03 & 1.2562 & 1.3515 & 2.0971 & 3.0666 & 3.3306 \\
\hline & & 0.05 & 1.3414 & 1.7711 & 2.9406 & 3.3662 & 4.0339 \\
\hline & & 0.07 & 1.4386 & 2.1640 & 3.2621 & 3.7676 & 4.0941 \\
\hline & & 0.09 & 1.5409 & 2.4924 & 3.2907 & 4.1107 & 4.2912 \\
\hline & & 0.11 & 1.6405 & 2.7525 & 3.3018 & 4.1242 & 4.6963 \\
\hline & & 0.03 & 0.9658 & 1.2344 & 1.8146 & 2.0492 & 2.8447 \\
\hline & \multirow{4}{*}{$\mathrm{C}-\mathrm{SD}$} & 0.05 & 1.0103 & 1.5469 & 2.0032 & 2.6846 & 3.5870 \\
\hline & & 0.07 & 1.0687 & 1.8088 & 2.0913 & 3.3260 & 3.6433 \\
\hline & & 0.09 & 1.1345 & 1.9096 & 2.3052 & 3.5433 & 3.9864 \\
\hline & & 0.11 & 1.2024 & 1.9376 & 2.5488 & 3.5665 & 4.4067 \\
\hline & \multirow{5}{*}{ C-E1 } & 0.03 & 1.0001 & 1.3483 & 1.9236 & 2.1507 & 3.0996 \\
\hline & & 0.05 & 1.0721 & 1.7532 & 2.0238 & 2.9514 & 3.6172 \\
\hline & & 0.07 & 1.1607 & 1.9669 & 2.2262 & 3.4814 & 3.8102 \\
\hline & & 0.09 & 1.2509 & 2.0039 & 2.5452 & 3.5550 & 4.2877 \\
\hline & & 0.11 & 1.3335 & 2.0281 & 2.8156 & 3.5706 & 4.6856 \\
\hline
\end{tabular}

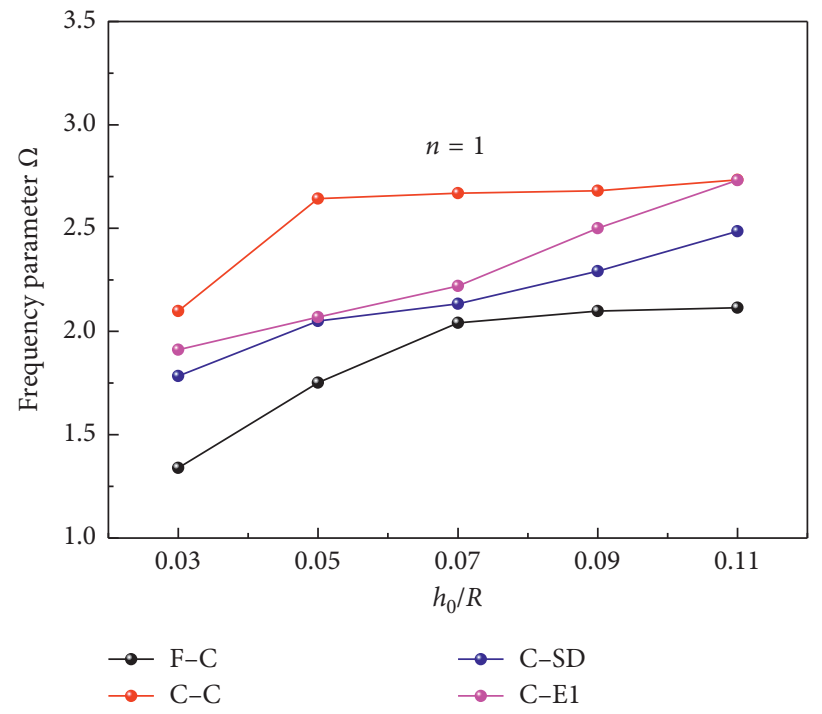

(a)

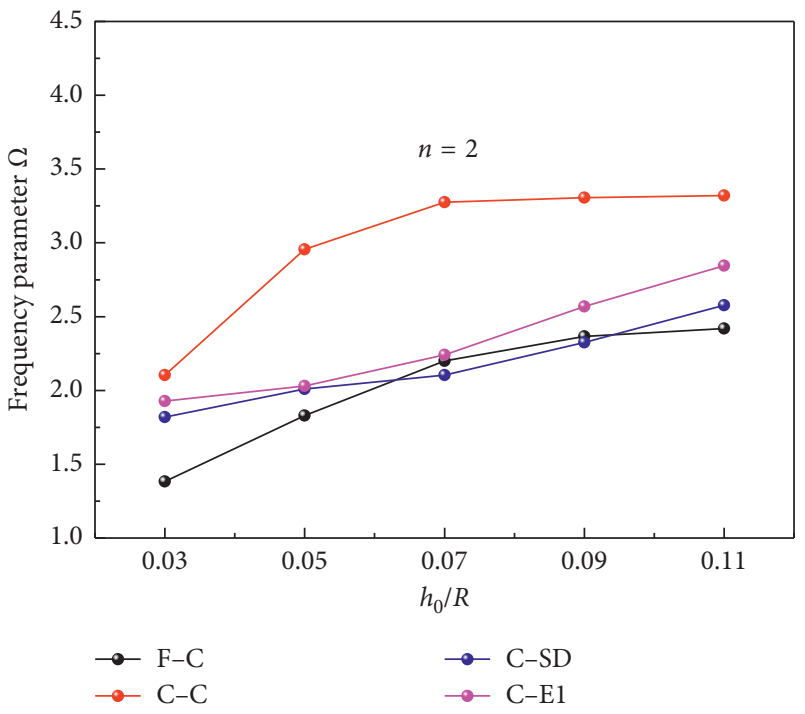

(b)

Figure 9: Continued. 


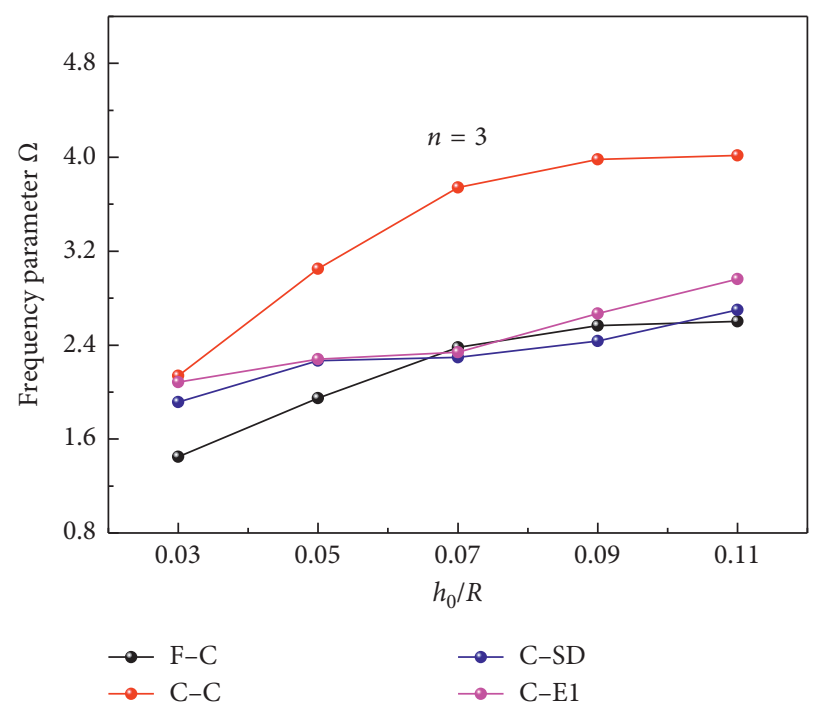

(c)

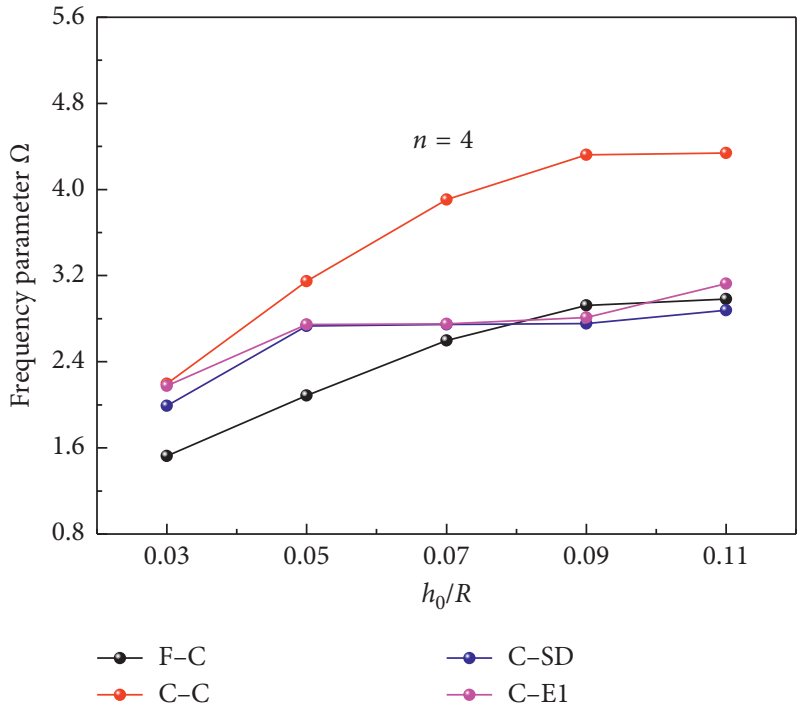

(d)

FIGURE 9: Frequency parameters $\Omega$ of FG spherical shell with uniform variable thickness under different $h_{0} / R$ ratio $(m=3)$.

spherical torus with uniform variable thickness under different $h_{0} / R$ ratios. Not unexpectedly, the results of the structure tend to rise with $h_{0} / R$ increasing from the examples. In addition, as described in the previous analysis, the frequency parameters can be obviously influenced by different edge restraints.

\section{Conclusions}

The aim of this study is to establish a unified semianalytical approach to carry out the free vibration solutions of FG spherical torus with uniform variable thickness along axial direction under general boundary conditions based on Ritz method. The first-order shear deformation theory is employed to formulate the analytical model. The spherical torus structure is artificially divided into proper shell segments to satisfy the computing requirement of high-order vibration responses according to domain decomposition method. The two adjacent segments are connected by the artificial springs, and the continuity condition and different boundary conditions can be obtained by assigning the appropriate values of springs. The displacement functions components are double-mixed series, in which Fourier series and unified Jacobi polynomials, respectively, represent displacement function along circumferential direction and axial direction. Then the Ritz method is used to obtain final solutions. The numerical results obtained by the proposed method show great agreement with FEM and those in the previously published literatures. In addition, the effects of boundary conditions and geometric parameters on the vibration responses of the structure are also presented. The most novelty of this paper is the unified Jacobi polynomial which can generalize the selection of admissible displacement functions. Therefore, the work of this paper can be used as a basic data to provide reference for future staff of such research.

\section{Appendix}

The generalized mass and stiffness matrix of FG spherical torus structure used in equation (22) are given as

$$
\begin{aligned}
& M=\operatorname{diag}\left[M^{1}, M^{2}, \ldots, M^{H}\right], \\
& \mathbf{M}^{i}=\int_{\theta_{i}}^{\theta_{i+1}} \int_{0}^{2 \pi}\left[\begin{array}{ccccc}
\mathbf{M}_{u u} & 0 & 0 & \mathbf{M}_{u \theta} & 0 \\
0 & \mathbf{M}_{v v} & 0 & 0 & \mathbf{M}_{v \varphi} \\
0 & 0 & \mathbf{M}_{w w} & 0 & 0 \\
\mathbf{M}_{u \theta} & 0 & 0 & \mathbf{M}_{\theta \theta} & 0 \\
0 & \mathbf{M}_{v \varphi} & 0 & 0 & \mathbf{M}_{\varphi \varphi}
\end{array}\right] A B \mathrm{~d} \theta \mathrm{d} \varphi,
\end{aligned}
$$




$$
\begin{aligned}
& \mathbf{M}_{u u}=I_{0} \mathbf{U}^{T} \mathbf{U}, \\
& \mathbf{M}_{v v}=I_{0} \mathbf{V}^{T} \mathbf{V}, \\
& \mathbf{M}_{w w}=I_{0} \mathbf{W}^{T} \mathbf{W}, \\
& \mathbf{M}_{x x}=I_{2} \Theta^{T} \boldsymbol{\Theta}, \\
& \mathbf{M}_{\varphi \varphi}=I_{2} \boldsymbol{\Phi}^{T} \boldsymbol{\Phi}, \\
& \mathbf{M}_{u \theta}=I_{1} \mathbf{U}^{T} \boldsymbol{\Theta}, \\
& \mathbf{M}_{v \varphi}=I_{1} \mathbf{V}^{T} \boldsymbol{\Phi}, \\
& \mathbf{U}=\mathbf{P}_{m} \otimes \mathbf{C}_{n}, \\
& \mathbf{V}=\mathbf{P}_{m} \otimes \mathbf{S}_{n}, \\
& \mathbf{W}=\mathbf{P}_{m} \otimes \mathbf{C}_{n}, \\
& \Theta=\mathbf{P}_{m} \otimes \mathbf{C}_{n}, \\
& \Phi=\mathbf{P}_{m} \otimes \mathbf{S}_{n}, \\
& \mathbf{P}_{m}=\left[P_{0}^{(\alpha, \beta)}(\theta), P_{1}^{(\alpha, \beta)}(\theta), \ldots, P_{m}^{(\alpha, \beta)}(\theta), \ldots, P_{M}^{(\alpha, \beta)}(\theta)\right], \\
& \mathbf{C}_{n}=[\cos (0 \varphi), \cos (1 \varphi), \ldots, \cos (n \varphi), \ldots, \cos (N \varphi)], \\
& \mathbf{S}_{n}=[\sin (0 \varphi), \sin (1 \varphi), \ldots, \sin (n \varphi), \ldots, \sin (N \varphi)], \\
& \mathbf{K}=\mathbf{K}_{\xi}+\mathbf{K}_{b}+\mathbf{K}_{s} \text {, } \\
& \mathbf{K}_{\xi}=\operatorname{diag}\left[\mathbf{K}^{\mathbf{1}}, \mathbf{K}^{\mathbf{2}}, \ldots, \mathbf{K}^{\mathrm{H}}\right] \text {, } \\
& \mathbf{K}^{i}=\int_{\theta_{i}}^{\theta_{i+1}} \int_{0}^{2 \pi}\left[\begin{array}{ccccc}
\mathbf{K}_{u u} & \mathbf{K}_{u v} & \mathbf{K}_{u w} & \mathbf{K}_{u \theta} & \mathbf{K}_{u \varphi} \\
\mathbf{K}_{u v}^{T} & \mathbf{K}_{v v} & \mathbf{K}_{v w} & \mathbf{K}_{v \theta} & \mathbf{K}_{v \varphi} \\
\mathbf{K}_{u w}^{T} & \mathbf{K}_{v w}^{T} & \mathbf{K}_{w w} & \mathbf{K}_{w \theta} & \mathbf{K}_{w \varphi} \\
\mathbf{K}_{u \theta}^{T} & \mathbf{K}_{v \theta}^{T} & \mathbf{K}_{w \theta}^{T} & \mathbf{K}_{\theta \theta} & \mathbf{K}_{\theta \varphi} \\
\mathbf{K}_{u \varphi}^{T} & \mathbf{K}_{v \varphi}^{T} & \mathbf{K}_{w \varphi}^{T} & \mathbf{K}_{\theta \varphi}^{T} & \mathbf{K}_{\varphi \varphi}
\end{array}\right] A B \mathrm{~d} \theta \mathrm{d} \varphi, \\
& \mathbf{K}_{\xi, u u}=A_{11} \frac{1}{R^{2}} \frac{\partial \mathbf{U}^{T}}{\partial \theta} \frac{\partial \mathbf{U}}{\partial \theta}+A_{11} \frac{1}{R^{2} T_{\theta}^{2}} \mathbf{U}^{T} \mathbf{U}+A_{66} \frac{1}{R^{2} S_{\theta}^{2}} \frac{\partial \mathbf{U}^{T}}{\partial \varphi} \frac{\partial \mathbf{U}}{\partial \varphi}+A_{12} \frac{1}{R^{2} T_{\theta}}\left(\frac{\partial \mathbf{U}^{T}}{\partial \theta} \mathbf{U}+\mathbf{U}^{T} \frac{\partial \mathbf{U}}{\partial \theta}\right)+k_{\text {shear }} A_{66} \frac{1}{R^{2}} \mathbf{U}^{T} \mathbf{U}, \\
& \mathbf{K}_{\xi, u v}=\left(A_{11} \frac{C_{\theta}}{R^{2} S_{\theta}^{2}} \mathbf{U}+A_{12} \frac{1}{R^{2} S_{\theta}} \frac{\partial \mathbf{U}}{\partial \theta}\right)^{T} \frac{\partial \mathbf{V}}{\partial \varphi}+A_{66} \frac{1}{R S_{\theta}} \frac{\partial \mathbf{U}^{T}}{\partial \varphi} \mathbf{V}_{1}, \\
& \mathbf{K}_{\xi, u w}=\left[\left(A_{11} \frac{1}{R^{2}}+A_{12} \frac{1}{R^{2}}\right) \frac{\partial \mathbf{U}}{\partial \theta}+\left(A_{11} \frac{1}{R^{2} T_{\theta}}+A_{12} \frac{1}{R^{2} T_{\theta}}\right) \mathbf{U}\right]^{T} \mathbf{W}-k_{\text {shear }} A_{66} \frac{1}{R^{2}} \mathbf{U}^{T} \frac{\partial \mathbf{W}}{\partial \theta}, \\
& \mathbf{K}_{\xi, u \theta}=-k_{\text {shear }} A_{66} \frac{1}{R} \mathbf{U}^{T} \boldsymbol{\Theta}+B_{11} \frac{1}{R^{2}} \frac{\partial \mathbf{U}^{T}}{\partial \theta} \frac{\partial \Theta}{\partial \theta}+B_{12} \frac{1}{R^{2} T_{\theta}} \frac{\partial \mathbf{U}^{T}}{\partial \theta} \boldsymbol{\Theta}+B_{12} \frac{1}{R^{2} T_{\theta}} \mathbf{U}^{T} \frac{\partial \Theta}{\partial \theta}+B_{11} \frac{1}{R^{2} T_{\theta}^{2}} \mathbf{U}^{T} \boldsymbol{\Theta}+B_{66} \frac{1}{R^{2} S_{\theta}^{2}} \frac{\partial \mathbf{U}^{T}}{\partial \varphi} \frac{\partial \Theta}{\partial \varphi}, \\
& \mathbf{K}_{\xi, u \varphi}=B_{12} \frac{1}{R^{2} S_{\theta}} \frac{\partial \mathbf{U}^{T}}{\partial \theta} \frac{\partial \Phi}{\partial \varphi}+B_{11} \frac{1}{R^{2} T_{\theta} S_{\theta}} \mathbf{U}^{T} \frac{\partial \Phi}{\partial \varphi}+B_{66} \frac{1}{R S_{\theta}} \frac{\partial \mathbf{U}^{T}}{\partial \varphi} \boldsymbol{\Phi}_{1}, \\
& \mathbf{K}_{\xi, v v}=A_{11} \frac{1}{R^{2} S_{\theta}^{2}} \frac{\partial \mathbf{V}^{T}}{\partial \varphi} \frac{\partial \mathbf{V}}{\partial \varphi}+A_{66} \mathbf{V}_{1}^{T} \mathbf{V}_{1}+k_{\text {shear }} A_{66} \frac{1}{R^{2}} \mathbf{V}^{T} \mathbf{V}
\end{aligned}
$$




$$
\begin{aligned}
& \mathbf{K}_{\xi, v w}=\left(A_{11} \frac{1}{R^{2} S_{\theta}}+A_{12} \frac{1}{R^{2} S_{\theta}}\right) \frac{\partial \mathbf{V}^{T}}{\partial \varphi} \mathbf{W}-k_{\text {shear }} A_{66} \frac{1}{R^{2} S_{\theta}} \mathbf{V}^{T} \frac{\partial \mathbf{W}}{\partial \varphi}, \\
& \mathbf{K}_{\xi, \nu \theta}=B_{12} \frac{1}{R^{2} S_{\theta}} \frac{\partial \mathbf{V}^{T}}{\partial \varphi} \frac{\partial \Theta}{\partial \theta}+B_{11} \frac{1}{R^{2} T_{\theta} S_{\theta}} \frac{\partial \mathbf{V}^{T}}{\partial \varphi} \Theta+B_{66} \frac{1}{R S_{\theta}} \mathbf{V}_{1}^{T} \frac{\partial \Theta}{\partial \varphi}, \\
& \mathbf{K}_{\xi, \nu \varphi}=-k_{\text {shear }} A_{66} \frac{1}{R} \mathbf{V}^{T} \boldsymbol{\Phi}+B_{11} \frac{1}{R^{2} S_{\theta}^{2}} \frac{\partial \mathbf{V}^{T}}{\partial \varphi} \frac{\partial \Phi}{\partial \varphi}+B_{66} \frac{1}{R S_{\theta}} \mathbf{V}_{1}^{T} \boldsymbol{\Phi}_{1} \\
& \mathbf{K}_{\xi, w w}=\frac{2}{R^{2}}\left(A_{11}+A_{12}\right) \mathbf{W}^{T} \mathbf{W}+k_{\text {shear }} A_{66}\left(\frac{1}{R^{2}} \frac{\partial \mathbf{W}^{T}}{\partial \theta} \frac{\partial \mathbf{W}}{\partial \theta}+\frac{1}{R^{2} S_{\theta}^{2}} \frac{\partial \mathbf{W}^{T}}{\partial \varphi} \frac{\partial \mathbf{W}}{\partial \varphi}\right), \\
& \mathbf{K}_{\xi, w \theta}=k_{\text {shear }} A_{66} \frac{1}{R} \frac{\partial \mathbf{W}^{T}}{\partial \theta} \boldsymbol{\Theta}+B_{11} \frac{1}{R^{2}} \mathbf{W}^{T} \frac{\partial \Theta}{\partial \theta}+B_{12} \frac{1}{R^{2} T_{\theta}} \mathbf{W}^{T} \boldsymbol{\Theta}+B_{12} \frac{1}{R^{2}} \mathbf{W}^{T} \frac{\partial \Theta}{\partial \theta}+B_{11} \frac{1}{R^{2} T_{\theta}} \mathbf{W}^{T} \boldsymbol{\Theta}, \\
& \mathbf{K}_{\xi, w \varphi}=k_{\text {shear }} A_{66} \frac{1}{R S_{\theta}} \frac{\partial \mathbf{W}^{T}}{\partial \varphi} \Phi+B_{12} \frac{1}{R^{2} S_{\theta}} \mathbf{W}^{T} \frac{\partial \Phi}{\partial \varphi}+B_{11} \frac{1}{R^{2} S_{\theta}} \mathbf{W}^{T} \frac{\partial \Phi}{\partial \varphi}, \\
& \mathbf{K}_{\xi, \theta \theta}=k_{\text {shear }} A_{66} \Theta^{T} \Theta+D_{11} \frac{1}{R^{2}} \frac{\partial \Theta^{T}}{\partial \theta} \frac{\partial \Theta}{\partial \theta}+D_{11} \frac{1}{R^{2} T_{\theta}^{2}} \Theta^{T} \Theta+D_{66} \frac{1}{R^{2} S_{\theta}^{2}} \frac{\partial \Theta^{T}}{\partial \varphi} \frac{\partial \Theta}{\partial \varphi}+D_{12} \frac{1}{R^{2} T_{\theta}}\left(\Theta^{T} \frac{\partial \Theta}{\partial \theta}+\frac{\partial \Theta^{T}}{\partial \theta} \Theta\right), \\
& \mathbf{K}_{\xi, \theta \varphi}=D_{11} \frac{1}{R^{2} T_{\theta} S_{\theta}} \boldsymbol{\Theta}^{T} \frac{\partial \Phi}{\partial \varphi}+D_{66} \frac{1}{R S_{\theta}} \frac{\partial \Theta^{T}}{\partial \varphi} \boldsymbol{\Phi}_{1}+D_{12} \frac{1}{R^{2} S_{\theta}} \frac{\partial \Theta^{T}}{\partial \theta} \frac{\partial \Phi}{\partial \varphi}, \\
& \mathbf{K}_{\xi, \varphi \varphi}=k_{\text {shear }} A_{66} \boldsymbol{\Phi}^{T} \boldsymbol{\Phi}+D_{11} \frac{1}{R^{2} S_{\theta}^{2}} \frac{\partial \boldsymbol{\Phi}^{T}}{\partial \varphi} \frac{\partial \boldsymbol{\Phi}}{\partial \varphi}+D_{66} \boldsymbol{\Phi}_{1}^{T} \boldsymbol{\Phi}_{1},
\end{aligned}
$$

where

$$
\begin{aligned}
C_{\theta} & =\cos (\theta), \\
S_{\theta} & =\sin (\theta), \\
T_{\theta} & =\tan (\theta), \\
\mathbf{V}_{1} & =\frac{1}{R} \frac{\partial \mathbf{V}}{\partial \theta}-\frac{1}{R T_{\theta}} \mathbf{V}, \\
\boldsymbol{\Phi}_{1} & =\frac{1}{R} \frac{\partial \Phi}{\partial \theta}-\frac{1}{R T_{\theta}} \mathbf{\Phi}, \\
\mathbf{K}_{b} & =\operatorname{diag}\left[\mathbf{K}_{b l}, 0, \ldots, \mathbf{K}_{b r}\right], \\
\mathbf{K}_{b l} & =\int_{0}^{2 \pi} \operatorname{diag}\left[\mathbf{K}_{b l, u u}, \mathbf{K}_{b l, v v}, \mathbf{K}_{b l, w w}, \mathbf{K}_{b l, \theta \theta}, \mathbf{K}_{b l, \varphi \varphi}\right]_{\theta_{\xi}=\theta_{0}} B \mathrm{~d} \varphi, \\
\mathbf{K}_{b l, u u} & =k_{u, 0} \mathbf{U}^{T} \mathbf{U}, \\
\mathbf{K}_{b l, v v} & =k_{v, 0} \mathbf{V}^{T} \mathbf{V}, \\
\mathbf{K}_{b l, w w} & =k_{w, 0} \mathbf{W}^{T} \mathbf{W}, \\
\mathbf{K}_{b l, \theta \theta} & =k_{\theta, 0} \boldsymbol{\Theta}^{T} \boldsymbol{\Theta}, \\
\mathbf{K}_{b l, \varphi \varphi} & =k_{\varphi, 0} \boldsymbol{\Phi}^{T} \boldsymbol{\Phi},
\end{aligned}
$$




$$
\begin{aligned}
& \mathbf{K}_{b r}=\int_{0}^{2 \pi} \operatorname{diag}\left[\mathbf{K}_{b r, u u}, \mathbf{K}_{b r, v v}, \mathbf{K}_{b r, w w}, \mathbf{K}_{b r, \theta \theta}, \mathbf{K}_{b r, \varphi \varphi}\right]_{\theta_{\xi}=\theta_{1}} B \mathrm{~d} \varphi \\
& \mathbf{K}_{b r, u u}=k_{u, 1} \mathbf{U}^{T} \mathbf{U} \\
& \mathbf{K}_{b r, v v}=k_{v, 1} \mathbf{V}^{T} \mathbf{V} \text {, } \\
& \mathbf{K}_{b r, w w}=k_{w, 1} \mathbf{W}^{T} \mathbf{W} \text {, } \\
& \mathbf{K}_{b r, \theta \theta}=k_{\theta, 1} \Theta^{T} \Theta, \\
& \mathbf{K}_{b r, \varphi \varphi}=k_{\varphi, 1} \boldsymbol{\Phi}^{T} \boldsymbol{\Phi}, \\
& \mathbf{K}_{s}=\operatorname{diag}\left[\mathbf{K}_{s}^{1}, \mathbf{K}_{s}^{2}, \ldots, \mathbf{K}_{s}^{H}\right], \\
& \mathbf{K}_{s}^{i}=\int_{0}^{2 \pi}\left[\begin{array}{ll}
\mathbf{K}_{s 0} & \mathbf{K}_{s 1} \\
\mathbf{K}_{s 1}^{T} & \mathbf{K}_{s 2}
\end{array}\right] B \mathrm{~d} \varphi, \\
& \mathbf{K}_{s 0}=\operatorname{diag}\left[\mathbf{K}_{u_{i} u_{i}}, \mathbf{K}_{v_{i} v_{i}}, \mathbf{K}_{w_{i} w_{i}}, \mathbf{K}_{\theta_{i} \theta_{i}}, \mathbf{K}_{\varphi_{i} \varphi_{i}}\right] \text {, } \\
& \mathbf{K}_{s 1}=\operatorname{diag}\left[\mathbf{K}_{u_{i} u_{i+1}}, \mathbf{K}_{v_{i} v_{i+1}}, \mathbf{K}_{w_{i} w_{i+1}}, \mathbf{K}_{\theta_{i} \theta_{i+1}}, \mathbf{K}_{\varphi_{i} \varphi_{i+1}}\right] \text {, } \\
& \mathbf{K}_{s 2}=\operatorname{diag}\left[\mathbf{K}_{u_{i+1} u_{i+1}}, \mathbf{K}_{v_{i+1} v_{i+1}}, \mathbf{K}_{w_{i+1} w_{i+1}}, \mathbf{K}_{\theta_{i+1} \theta_{i+1}}, \mathbf{K}_{\varphi_{i+1} \varphi_{i+1}}\right] \text {, } \\
& \mathbf{K}_{u_{i} u_{i}}=k_{u} \mathbf{U}_{i}^{T} \mathbf{U}_{i}, \\
& \mathbf{K}_{v_{i} v_{i}}=k_{v} \mathbf{V}_{i}^{T} \mathbf{V}_{i} \text {, } \\
& \mathbf{K}_{w_{i} w_{i}}=k_{w} \mathbf{W}_{i}^{T} \mathbf{W}_{i}, \\
& \mathbf{K}_{u_{i} u_{i+1}}=-k_{u} \mathbf{U}_{i}^{T} \mathbf{U}_{i+1}, \\
& \mathbf{K}_{v_{i} v_{i+1}}=-k_{v} \mathbf{V}_{i}^{T} \mathbf{V}_{i+1} \text {, } \\
& \mathbf{K}_{w_{i} w_{i+1}}=-k_{w} \mathbf{W}_{i}^{T} \mathbf{W}_{i+1}, \\
& \mathbf{K}_{\theta_{i} \theta_{i+1}}=-k_{\theta} \boldsymbol{\Theta}_{i}^{T} \boldsymbol{\Theta}_{i+1}, \\
& \mathbf{K}_{\varphi_{i} \varphi_{i+1}}=-k_{\varphi} \boldsymbol{\Phi}_{i}^{T} \boldsymbol{\Phi}_{i+1}, \\
& \mathbf{K}_{u_{i+1} u_{i+1}}=k_{u} \mathbf{U}_{i+1}^{T} \mathbf{U}_{i+1}, \\
& \mathbf{K}_{v_{i+1} v_{i+1}}=k_{v} \mathbf{V}_{i+1}^{T} \mathbf{V}_{i+1} \text {, } \\
& \mathbf{K}_{w_{i+1} w_{i+1}}=k_{w} \mathbf{W}_{i+1}^{T} \mathbf{W}_{i+1}, \\
& \mathbf{K}_{\theta_{i+1} \theta_{i+1}}=k_{\theta} \boldsymbol{\Theta}_{i+1}^{T} \boldsymbol{\Theta}_{i+1}, \\
& \mathbf{K}_{\varphi_{i+1} \varphi_{i+1}}=k_{\varphi} \boldsymbol{\Phi}_{i+1}^{T} \boldsymbol{\Phi}_{i+1}, \\
& \mathbf{K}_{\theta_{i} \theta_{i}}=k_{\theta} \boldsymbol{\Theta}_{i}^{T} \boldsymbol{\Theta}_{i}, \\
& \mathbf{K}_{\varphi_{i} \varphi_{i}}=k_{\varphi} \boldsymbol{\Phi}_{i}^{T} \boldsymbol{\Phi}_{i},
\end{aligned}
$$




\section{Data Availability}

The data used to support the findings of this study are included within the article.

\section{Conflicts of Interest}

The authors declare that there are no conflicts of interest regarding the publication of this paper.

\section{Acknowledgments}

This study was funded by the National Natural Science Foundation of China (nos. 51209052 and 51709063), National key Research and Development program (2016YFC0303406), Fundamental Research Funds for the Central University (HEUCFD1515 and HEUCFM170113), Assembly Advanced Research Fund of China (6140210020105), Naval preresearch project, China Postdoctoral Science Foundation (2014M552661), and Ph.D. Student Research and Innovation Fund of the Fundamental Research Funds for the Central Universities (HEUGIP201801).

\section{References}

[1] M. Koizumi, "FGM activities in Japan," Composites Part B: Engineering, vol. 28, no. 1-2, pp. 1-4, 1997.

[2] F. Tornabene, "2-D GDQ solution for free vibrations of anisotropic doubly-curved shells and panels of revolution," Composite Structures, vol. 93, no. 7, pp. 1854-1876, 2011.

[3] F. Tornabene, A. Liverani, and G. Caligiana, "General anisotropic doubly-curved shell theory: a differential quadrature solution for free vibrations of shells and panels of revolution with a free-form meridian," Journal of Sound and Vibration, vol. 331, no. 22, pp. 4848-4869, 2012.

[4] F. Tornabene, S. Brischetto, N. Fantuzzi, and E. Viola, "Numerical and exact models for free vibration analysis of cylindrical and spherical shell panels," Composites Part B: Engineering, vol. 81, pp. 231-250, 2015.

[5] F. Tornabene and E. Viola, "Vibration analysis of spherical structural elements using the GDQ method," Computers and Mathematics with Applications, vol. 53, no. 10, pp. 1538-1560, 2007.

[6] H. Li, F. Pang, Q. Gong, and Y. Teng, "Free vibration analysis of axisymmetric functionally graded doubly-curved shells with un-uniform thickness distribution based on Ritz method," Composite Structures, vol. 225, p. 111145, 2019.

[7] F. Pang, H. Li, X. Wang, X. Miao, and S. Li, "A semi analytical method for the free vibration of doubly-curved shells of revolution," Computers and Mathematics with Applications, vol. 75, no. 9, pp. 3249-3268, 2018.

[8] H. Li, F. Pang, and H. Chen, "A semi-analytical approach to analyze vibration characteristics of uniform and stepped annular-spherical shells with general boundary conditions," European Journal of Mechanics-A/Solids, vol. 74, pp. 48-65, 2019.

[9] H. Li, F. Pang, X. Miao, Y. Du, and H. Tian, "A semi-analytical method for vibration analysis of stepped doubly-curved shells of revolution with arbitrary boundary conditions," ThinWalled Structures, vol. 129, pp. 125-144, 2018.
[10] S. Hosseini-Hashemi and M. Fadaee, "On the free vibration of moderately thick spherical shell panel-A new exact closedform procedure," Journal of Sound and Vibration, vol. 330, no. 17, pp. 4352-4367, 2011.

[11] V. A. Polyakov, R. P. Shlitsa, V. V. Khitrov, and V. I. Zhigun, "An applied model for free radial vibrations of a closed spherical sandwich shell," Mechanics of Composite Materials, vol. 43, no. 4, pp. 331-344, 2007.

[12] M. Menaa and A. A. Lakis, "Free vibration of spherical shells using a hybrid finite element method," International Journal of Structural Stability and Dynamics, vol. 15, no. 4, 2015.

[13] V. K. Singh and S. K. Panda, "Large amplitude free vibration analysis of laminated composite spherical shells embedded with piezoelectric layers," Smart Structures and Systems, vol. 16, no. 5, pp. 853-872, 2015.

[14] V. K. Singh and S. K. Panda, "Linear static and free vibration analyses of laminated composite spherical shells," in Proceedings of the ASME Gas Turbine India Conference, Bangalore, India, December 2013.

[15] T. R. Mahapatra and S. K. Panda, "Nonlinear free vibration analysis of laminated composite spherical shell panel under elevated hygrothermal environment: a micromechanical approach," Aerospace Science and Technology, vol. 49, pp. 276288, 2016.

[16] S. K. Panda and B. N. Singh, "Nonlinear free vibration analysis of thermally post-buckled composite spherical shell panel," International Journal of Mechanics and Materials in Design, vol. 6, no. 2, pp. 175-188, 2010.

[17] S. K. Panda and B. N. Singh, "Nonlinear free vibration of spherical shell panel using higher order shear deformation theory-a finite element approach," International Journal of Pressure Vessels and Piping, vol. 86, no. 6, pp. 373-383, 2009.

[18] Z. Su, G. Jin, S. Shi, and T. Ye, "A unified accurate solution for vibration analysis of arbitrary functionally graded spherical shell segments with general end restraints," Composite Structures, vol. 111, pp. 271-284, 2014.

[19] Q. Wang, F. Pang, B. Qin, and Q. Liang, “A unified formulation for free vibration of functionally graded carbon nanotube reinforced composite spherical panels and shells of revolution with general elastic restraints by means of the Rayleigh-Ritz method," Polymer Composites, vol. 39, no. S2, pp. E924-E944, 2018.

[20] Q. Wang, X. Cui, B. Qin, Q. Liang, and J. Tang, “A semianalytical method for vibration analysis of functionally graded (FG) sandwich doubly-curved panels and shells of revolution," International Journal of Mechanical Sciences, vol. 134, pp. 479-499, 2017.

[21] Y. Qu, X. Long, G. Yuan, and G. Meng, “A unified formulation for vibration analysis of functionally graded shells of revolution with arbitrary boundary conditions," Composites Part B: Engineering, vol. 50, pp. 381-402, 2013.

[22] H. Li, F. Pang, Y. Li, and C. Gao, "Application of first-order shear deformation theory for the vibration analysis of functionally graded doubly-curved shells of revolution," Composite Structures, vol. 212, pp. 22-42, 2019.

[23] H. Li, F. Pang, X. Wang, Y. Du, and H. Chen, "Free vibration analysis for composite laminated doubly-curved shells of revolution by a semi analytical method," Composite Structures, vol. 201, pp. 86-111, 2018.

[24] H. Li, F. Pang, H. Chen, and Y. Du, "Vibration analysis of functionally graded porous cylindrical shell with arbitrary boundary restraints by using a semi analytical method," Composites Part B: Engineering, vol. 164, pp. 249-264, 2019. 
[25] F. Tornabene and E. Viola, "Free vibrations of four-parameter functionally graded parabolic panels and shells of revolution," European Journal of Mechanics-A/Solids, vol. 28, no. 5, pp. 991-1013, 2009.

[26] J. N. Reddy and Z.-Q. Cheng, "Frequency correspondence between membranes and functionally graded spherical shallow shells of polygonal planform," International Journal of Mechanical Sciences, vol. 44, no. 5, pp. 967-985, 2002.

[27] A. M. A. Neves, A. J. M. Ferreira, E. Carrera et al., "Free vibration analysis of functionally graded shells by a higherorder shear deformation theory and radial basis functions collocation, accounting for through-the-thickness deformations," European Journal of Mechanics-A/Solids, vol. 37, pp. 24-34, 2013.

[28] V. R. Kar and S. K. Panda, "Free vibration responses of functionally graded spherical shell panels using finite element method," in Proceedings of the ASME Gas Turbine India Conference, Bangalore, India, December 2013.

[29] N. K. D. El-Kaabazi and D. Kennedy, "Calculation of natural frequencies and vibration modes of variable thickness cylindrical shells using the Wittrick-Williams algorithm," Computers and Structures, vol. 104-105, pp. 4-12, 2012.

[30] M. Liu, J. Liu, and Y. S. Cheng, "Free vibration of a fluid loaded ring-stiffened conical shell with variable thickness," Journal of Vibration and Acoustics-Transactions of the Asme, vol. 136, no. 5, 2014.

[31] Y. G. Qu, Y. Chen, Y. Chen, X. Long, H. Hua, and G. Meng, “A domain decomposition method for vibration analysis of conical shells with uniform and stepped thickness," Journal of Vibration and Acoustics-Transactions of the Asme, vol. 135, no. 1, 2013.

[32] Y. Qu, Y. Chen, X. Long, H. Hua, and G. Meng, "Free and forced vibration analysis of uniform and stepped circular cylindrical shells using a domain decomposition method," Applied Acoustics, vol. 74, no. 3, pp. 425-439, 2013.

[33] N. Wei, Z. S. Dongsheng, and J. L. Jiling, "Free vibration analysis of stiffened conical shell with variable thickness distribution," International Conference Machinery, Electronics and Control Simulation, vol. 614, pp. 7-11, 2014.

[34] J. H. Kang and A. W. Leissa, "Free vibration analysis of complete paraboloidal shells of revolution with variable thickness and solid paraboloids from a three-dimensional theory," Computers and Structures, vol. 83, no. 31-32, pp. 2594-2608, 2005.

[35] H. J. Xiang and J. Yang, "Free and forced vibration of a laminated FGM Timoshenko beam of variable thickness under heat conduction," Composites Part B: Engineering, vol. 39, no. 2, pp. 292-303, 2008.

[36] W. Jiang and D. Redekop, "Static and vibration analysis of orthotropic toroidal shells of variable thickness by differential quadrature," Thin-Walled Structures, vol. 41, no. 5, pp. 461-478, 2003.

[37] M. Z. Nejad, M. Jabbari, and M. Ghannad, "Elastic analysis of axially functionally graded rotating thick cylinder with variable thickness under non-uniform arbitrarily pressure loading," International Journal of Engineering Science, vol. 89, pp. 86-99, 2015.

[38] H. Li, F. Pang, X. Miao, and Y. Li, "Jacobi-Ritz method for free vibration analysis of uniform and stepped circular cylindrical shells with arbitrary boundary conditions: a unified formulation," Computers and Mathematics with Applications, vol. 77, no. 2, pp. 427-440, 2019.

[39] F. Pang, H. Li, J. Cui, Y. Du, and C. Gao, "Application of flügge thin shell theory to the solution of free vibration behaviors for spherical-cylindrical-spherical shell: a unified formulation," European Journal of Mechanics-A/Solids, vol. 74, pp. 381-393, 2019.

[40] F. Z. Pang, H. Li, F. Jing, and Y. Du, "Application of first-order shear deformation theory on vibration analysis of stepped functionally graded paraboloidal shell with general edge constraints," Materials, vol. 12, no. 1, p. 69, 2019.

[41] H. Li, F. Pang, X. Wang, Y. Du, and H. Chen, "Free vibration analysis of uniform and stepped combined paraboloidal, cylindrical and spherical shells with arbitrary boundary conditions," International Journal of Mechanical Sciences, vol. 145, pp. 64-82, 2018.

[42] A. H. Sofiyev, D. Hui, V. C. Haciyev et al., "The nonlinear vibration of orthotropic functionally graded cylindrical shells surrounded by an elastic foundation within first order shear deformation theory," Composites Part B: Engineering, vol. 116, pp. 170-185, 2017.

[43] H. Li, F. Pang, Y. Ren, X. Miao, and K. Ye, "Free vibration characteristics of functionally graded porous spherical shell with general boundary conditions by using first-order shear deformation theory," Thin-Walled Structures, vol. 144, Article ID 106331, 2019.

[44] F. Pang, H. Li, H. Chen, and Y. Shan, "Free vibration analysis of combined composite laminated cylindrical and spherical shells with arbitrary boundary conditions," Mechanics of Advanced Materials and Structures, pp. 1-18, 2019.

[45] F. Pang, C. Gao, J. Cui, Y. Ren, H. Li, and H. Wang, "A semianalytical approach for free vibration characteristics of functionally graded spherical shell based on first-order shear deformation theory," Shock and Vibration, vol. 2019, Article ID 7352901, 18 pages, 2019.

[46] H. Li, F. Pang, X. Miao, S. Gao, and F. Liu, "A semi analytical method for free vibration analysis of composite laminated cylindrical and spherical shells with complex boundary conditions," Thin-Walled Structures, vol. 136, pp. 200-220, 2019.

[47] A. H. Bhrawy, T. M. Taha, and J. A. Tenreiro Machado, "A review of operational matrices and spectral techniques for fractional calculus," Nonlinear Dynamics, vol. 81, no. 3, pp. 1023-1052, 2015. 


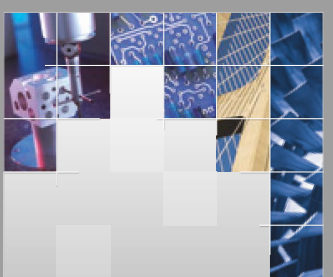

\section{Enfincering}
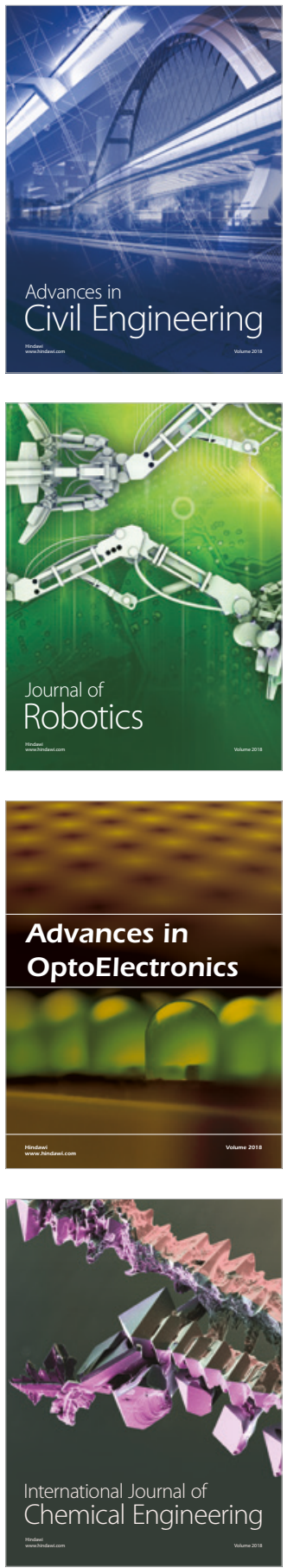

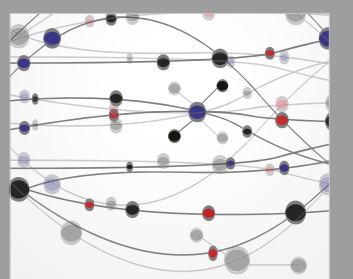

\section{Rotating \\ Machinery}

The Scientific World Journal

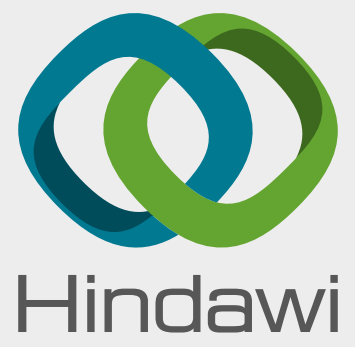

Submit your manuscripts at

www.hindawi.com
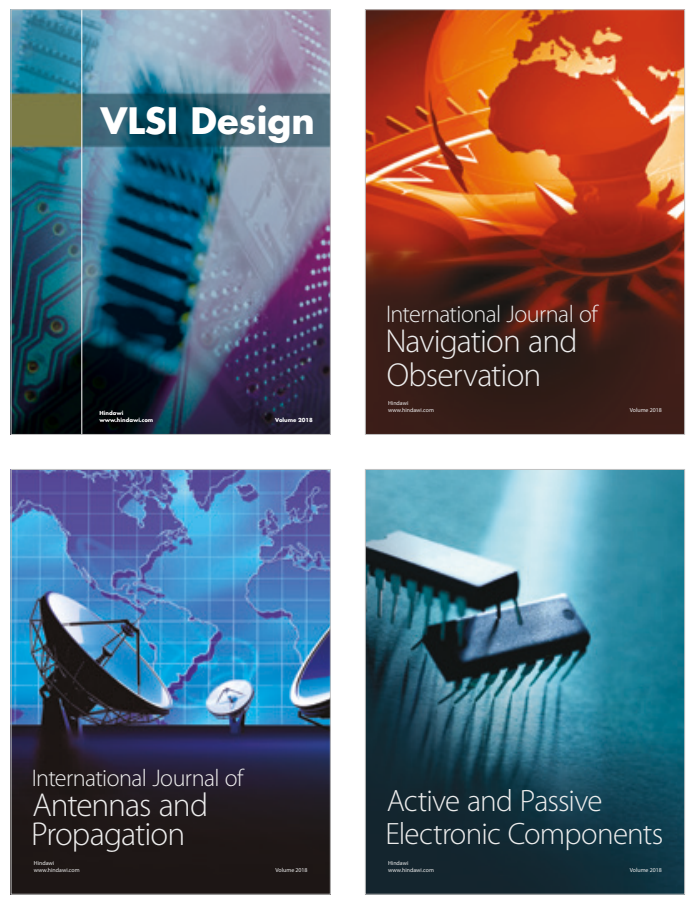
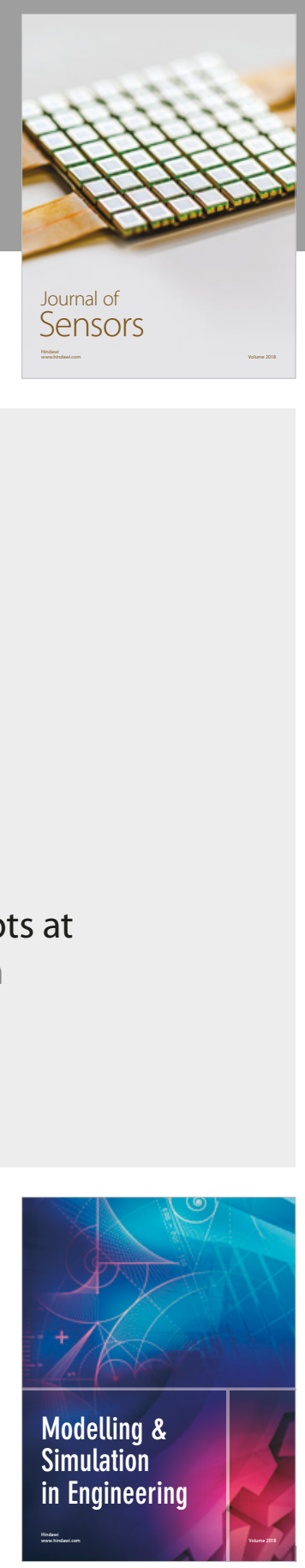

\section{Advances \\ Multimedia}
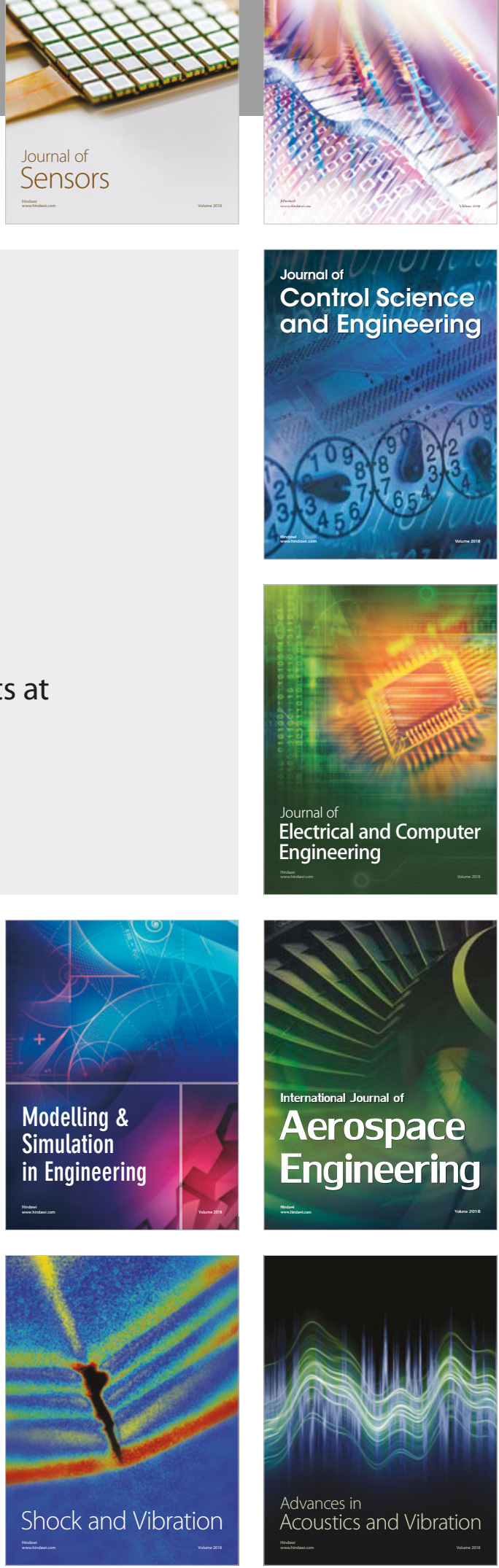